\title{
A Two-Phase Differential Evolution for Uniform Designs in Constrained Experimental Domains
}

\author{
Yong Wang, Member, IEEE, Biao Xu, Guangyong Sun, and Shengxiang Yang, Senior Member, IEEE
}

\begin{abstract}
In many real-world engineering applications, a uniform design needs to be conducted in a constrained experimental domain that includes linear/nonlinear and inequality/equality constraints. In general, these constraints make the constrained experimental domain small and irregular in the decision space. Therefore, it is difficult for current methods to produce a predefined number of samples and make the samples distribute uniformly in the constrained experimental domain. This paper presents a two-phase differential evolution for uniform designs in constrained experimental domains. In the first phase, considering the constraint violation as the fitness function, a clustering differential evolution is proposed to guide the population toward the constrained experimental domain from different directions promptly. As a result, a predefined number of samples can be obtained in the constrained experimental domain. In the second phase, maximizing the minimum Euclidean distance among samples is treated as another fitness function. By optimizing this fitness function, the samples produced in the first phase can be scattered uniformly in the constrained experimental domain. The performance of the proposed method has been tested and compared with another state-of-the-art method. Experimental results suggest that our method is significantly better than the compared method in the uniform designs of a new type of automotive crash box and five benchmark test problems. Moreover, the proposed method could be considered as a
\end{abstract}

Manuscript received July 4, 2016; revised November 4, 2016; accepted February 3, 2017. This work was supported in part by the Innovation-driven Plan in Central South University (No. 2015CXS012 and No. 2015CX007), in part by the National Natural Science Foundation of China under Grants 61673397, 61273314, and 61673331, in part by the EU Horizon 2020 Marie Sklodowska-Curie Individual Fellowships (Project ID: 661327), in part by the Engineering and Physical Sciences Research Council of UK under Grant EP/K001310/1, in part by the Hunan Provincial Natural Science Fund for Distinguished Young Scholars (Grant No. 2016JJ1018), in part by the Program for New Century Excellent Talents in University under Grant NCET-13-0596, and in part by State Key Laboratory of Advanced Design and Manufacturing for Vehicle Body. (Corresponding Author: Guangyong Sun)

Y. Wang is with the School of Information Science and Engineering, Central South University, Changsha 410083, China, and also with the Centre for Computational Intelligence (CCI), School of Computer Science and Informatics, De Montfort University, Leicester LE1 9BH, UK. (Email: ywang@csu.edu.cn)

B. $\mathrm{Xu}$ is with the School of Information Science and Engineering, Central South University, Changsha 410083, China. (Email: xubiao1189@163.com)

G. Sun is with the School of Aerospace, Mechanical and Mechatronic Engineering, Faculty of Engineering, University of Sydney, Sydney, NSW 2006, Australia, and also with the State Key Laboratory of Advanced Design and Manufacture for Vehicle Body, Hunan University, Changsha 410082, China. (Email: guangyong.sun@sydney.edu.au)

S. Yang is with the Centre for Computational Intelligence (CCI), School of Computer Science and Informatics, De Montfort University, Leicester LE1 9BH, UK. (Email: syang@dmu.ac.uk) general and promising framework for other uniform designs in constrained experimental domains.

Index Terms-A new type of automotive crash box, constrained experimental domain, differential evolution, uniform design.

\section{INTRODUCTION}

$\mathrm{E}$ NTERPRISES always try to provide high-quality, low-cost products and shorten lead time to survive in fierce competitive environments. This can be achieved through innovative design. Traditional product design is based on empirical and/or trial-and-error processes. Utilizing traditional methods to develop a new product will inevitably lead to a long design cycle and excessive costs because of numerous prototype tests. With the rapid development of advanced computer technology, finite element analysis has become a well-established numerical simulation tool to precisely predict product performance in the design stage, which can reduce design and prototyping costs [1]. Since the design scheme should be manually adjusted in line with engineers' experience, finite element analysis needs to be performed many times to reevaluate the results. Furthermore, the above process cannot guarantee the global optimum. In order to achieve more active design, finite element analysis-based optimization has been developed as a promising manner to systematically seek an optimal design [2]. Although finite element analysis-based optimization is able to improve the design level, it is essentially an iterative process. Note that computation-intensive design problems are becoming increasingly common in automotive, aerospace, transportation, and defense industries [3]. With respect to such design problems, it is time-consuming for one iteration. For example, in full vehicle crashworthiness, to meet all safety criteria, the design is intrinsically a daunting optimization task often involving multiple loading cases (e.g., frontal/side/rear impact, pedestrian safety, roof crush, interior head impact, rollover), multiple disciplines, and multiple objectives [4]-[6]. Just taking the computational cost into account, it has been reported that it takes Ford Motor Company about 36-160 hours to run one full vehicle crash simulation [7]. In general, a full vehicle crashworthiness design needs to call hundreds of simulations. In order to enhance the computational efficiency in design, highly accurate surrogate models have been widely used [8]-[10]. How to generate representative samples is the primary issue for constructing highly accurate surrogate models. The design of experiment can be considered to be an essential step toward this purpose [11].

The design of experiment aims to appropriately select a set of samples to provide uniform coverage over the experimental domain of interest [12]. Currently, the design of experiment has 


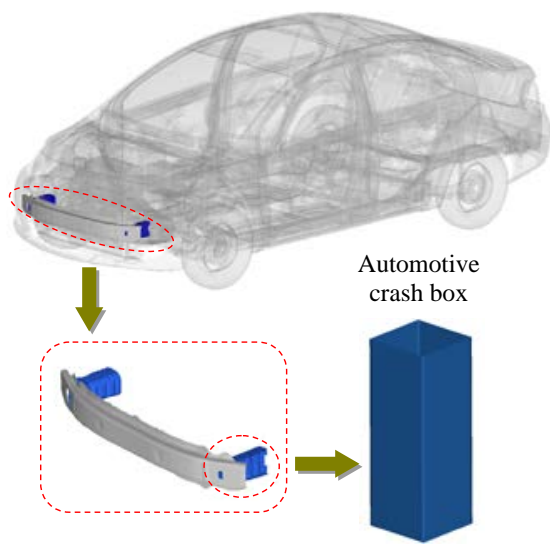

Fig. 1. The illustration of the automotive crash box

been broadly applied to computer experiments and industrial experiments [13]-[15]. Many techniques have been proposed for the design of experiment, such as orthogonal design [16]-[18], Latin square design [19]-[21], and uniform design [22]-[24]. Among them, uniform design is able to obtain more information from the experimental domain with a smaller number of samples [25]. Very often, for uniform designs of some complex practical situations, there are many linear/ nonlinear and inequality/equality constraints due to the interactions among the decision variables [26]. The uniform design of a new type of automotive crash box for lightweight can be taken as an example. The automotive crash box (as shown in Fig. 1) always bears very complex loading, which implies that different regions should have different roles to maximize the usage of materials. How to exhaust the potential of materials according to the performance requirements is one of the most important issues for automotive lightweight design. Fortunately, variable-thickness rolled blank can vary the blank thickness with a continuous thickness transition through adjusting the roll gap [27]. This innovative technology can easily realize the customized thickness of a new type of automotive crash box. Note that different thickness distributions of the variable-thickness rolled blanks lead to different performance; therefore, it is necessary to optimize the thickness distributions to maximize performance [28]. The optimal thickness distribution among different zones including the thin zones, thickness zones, and transition zones should satisfy a variety of manufacturing constraints [27], which are typical linear/nonlinear and inequality/equality constraints.

In this paper, the aim of a uniform design is to sample uniformly over a constrained experimental domain which is usually a small and irregular region in the decision space because of constraints. At present, many uniform design approaches have been proposed for this purpose, including:

1) Traditional uniform design methods: This kind of method firstly generates a number of uniform samples from the decision space, and then checks which samples satisfy all constraints, deleting the samples violating the constraints [29]-[32]. However, if there exist nonlinear constraints and/or equality constraints, the experimental domain is very small compared to the decision space. As a result, the samples satisfying all constraints are very scarce. Under this condition, it is a very challenging task for traditional uniform design methods to produce a desired number of samples.

2) Number-theoretic uniform design methods: This kind of method generates a number of uniform samples by number-theoretic techniques and tackles constraints by mapping function [33]-[35]. Unfortunately, this kind of method can only handle one linear equality constraint, and the other constraints are handled by the same process as in traditional uniform design methods. Hence, it faces substantial difficulties when dealing with complex nonlinear and/or equality constraints.

3) Heuristic uniform design methods: This kind of method models a uniform design in the constrained experimental domain as an optimization problem, and optimizes it via heuristic methods [36]-[39]. However, this kind of method is not suitable for uniform designs in the constrained experimental domains with high dimensionality and a large number of design points due to tremendous computational workload. Additionally, most methods do not introduce how to cope with nonlinear constraints and equality constraints.

From the above introduction, it is obvious that linear/ nonlinear and inequality/equality constraints pose a great challenge to current uniform design methods. Therefore, new insights toward uniform designs in constrained experimental domains are quite necessary. In this paper, inspired by [40], a two-phase differential evolution (DE) called ToPDE is proposed. In the first phase, a clustering DE integrated with constraint violation as the fitness function is presented to guide the population (i.e., a set of samples or a set of design points) toward the constrained experimental domain quickly. The advantages of the clustering DE are twofold. On one hand, it is capable of obtaining a predefined number of samples in the constrained experimental domain. On the other hand, it can keep the diversity of the population, with the purpose of providing high-quality candidate solutions for the second phase. In the second phase, another fitness function and a novel replacement strategy are proposed to make the population distribute uniformly in the constrained experimental domain, which can also dramatically reduce the computational time.

The main contributions of this paper can be summarized as follows:

1) ToPDE can be viewed as a new algorithmic framework with the incorporation of the properties of uniform designs in constrained experimental domains. Moreover, ToPDE is not dependent on the type and number of constraints.

2) Recognizing that the computational overhead of the existing heuristic uniform design methods is very high, the fitness function in the second phase offers a tradeoff between uniform distribution and computational cost.

3) ToPDE has been applied to the uniform designs of a practical engineering problem (i.e., a new type of automotive crash box) and five benchmark test problems. The experimental results show that ToPDE is significantly better than NTMD [33], which is a state- 


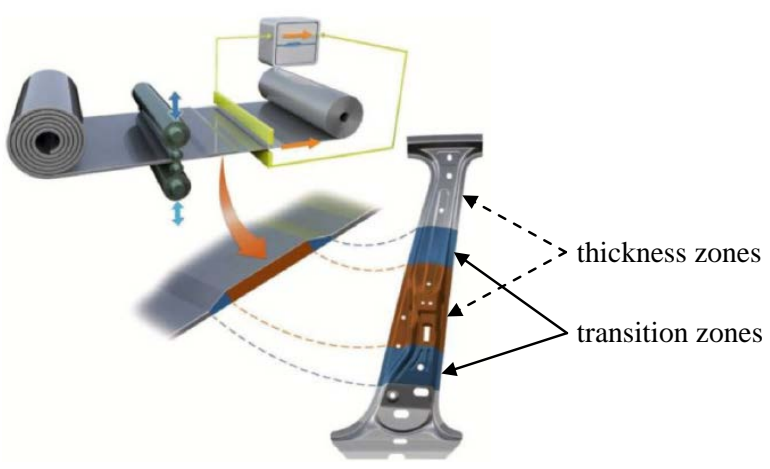

Fig. 2. The rolling processes of the variable-thickness rolled blank [27]

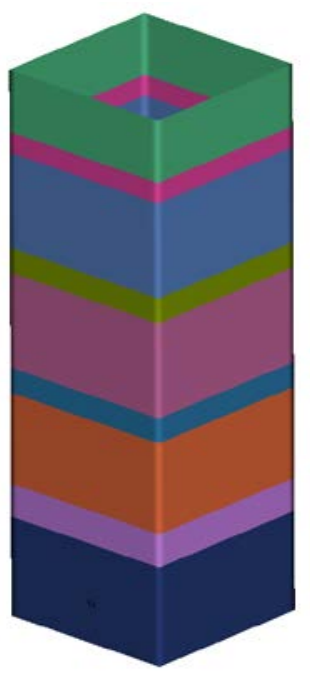

(a)

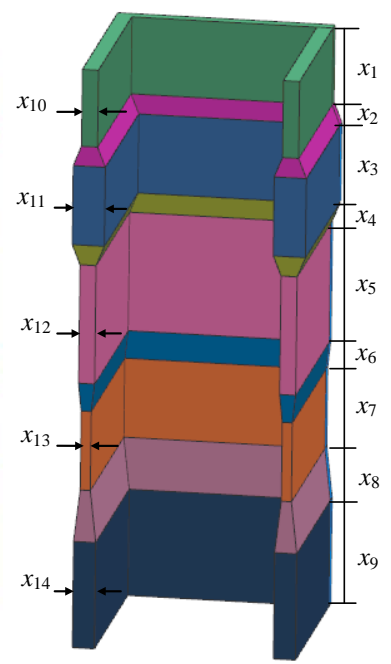

(b)
Fig. 3. The structure of a new type of automotive crash box. (a) The overall view of the new type of automotive crash box. (b) The sectional view of the new type of automotive crash box.

of-the-art number-theoretic method for uniform designs in constrained experimental domains.

This paper is organized as follows. Section II describes the uniform design of a new type of automotive crash box. Section III introduces the concepts of DE. ToPDE is proposed in Section IV. Section V presents the experimental results. Section VI further discusses the advantage and effectiveness of ToPDE. Finally, Section VII concludes this paper.

\section{The Uniform Design of A New Type of Automotive CRASH BOX}

Reducing the weight of an automobile is an effective method for saving energy and reducing emissions. The most important premise of automotive lightweight design is not to reduce product performance [41], especially safety. In all automotive safety parts, the crash box shown in Fig. 1 is an irreplaceable part [42] whose energy absorption has a significant effect on occupant safety. To improve the energy absorption, the most commonly used way is to increase the wall thickness of the traditional automotive crash box, which inevitably leads to a significant increase in weight. Therefore, it is very challenging to achieve light weight and high crashworthiness simultaneously. In order to address this issue, it is necessary to design
TABLE I

RELATIONSHIP OF THE DECISION VARIABLES IN THE NEW TYPE OF AUTOMOTIVE CRASH BOX

\begin{tabular}{lc}
\hline Relationship of the decision variables & Type of the constraints \\
\hline$x_{i}^{\text {lower }} \leq x_{i} \leq x_{i}^{\text {upper }}, i=1,2, \ldots, 14$ & Boundary constraints \\
\hline$\sum_{i=1}^{9} x_{i}=L$ & $\begin{array}{c}\text { Linear } \\
\text { equality } \\
\text { constraint }\end{array}$ \\
\hline$a \leq x_{10} / x_{11} \leq b, a \leq x_{11} / x_{12} \leq b$, & \\
$a \leq x_{12} / x_{13} \leq b, a \leq x_{13} / x_{14} \leq b$, & \\
$c \leq\left|x_{11}-x_{10}\right| / x_{2} \leq d, c \leq\left|x_{12}-x_{11}\right| / x_{4} \leq d$, & \\
$c \leq\left|x_{13}-x_{12}\right| / x_{6} \leq d, c \leq\left|x_{14}-x_{13}\right| / x_{8} \leq d$, & Nonlinear \\
$x_{1} x_{10}+0.5 x_{2}\left(x_{10}+x_{11}\right)+x_{3} x_{11}+$ & inequality \\
$0.5 x_{4}\left(x_{11}+x_{12}\right)+x_{5} x_{12}+0.5 x_{6}\left(x_{12}+x_{13}\right)+$ & constraints \\
$x_{7} x_{13}+0.5 x_{8}\left(x_{13}+x_{14}\right)+x_{9} x_{14} \leq \frac{M}{\rho l}$ & \\
$\rho$ denotes the density of material & \\
$l$ denotes the circumference of cross section & \\
\hline
\end{tabular}

TABLE II

Parameter Values of the NeW Type of Automotive Crash Box

\begin{tabular}{ll|ll}
\hline Parameter & Value & Parameter & Value \\
\hline$x_{1}^{\text {lower }}, \ldots, x_{9}^{\text {lower }}$ & $1(\mathrm{~mm})$ & $b$ & 1.5 \\
$x_{10}^{\text {lower }}, \ldots, x_{14}^{\text {lower }}$ & $0.8(\mathrm{~mm})$ & $c$ & $1 / 30$ \\
$x_{1}^{\text {upper }}, \ldots, x_{9}^{\text {upper }}$ & $175(\mathrm{~mm})$ & $d$ & $1 / 10$ \\
$x_{10}^{\text {upper }}, \ldots, x_{14}^{\text {upper }}$ & $2.5(\mathrm{~mm})$ & $M$ & $2.1168\left(K_{g}\right)$ \\
$L$ & $175(\mathrm{~mm})$ & $\rho$ & $2.7 \times 10^{-6}\left(\mathrm{~K}_{g} / \mathrm{mm}^{3}\right)$ \\
$a$ & 0.6 & $l$ & $320(\mathrm{~mm})$ \\
\hline
\end{tabular}

a new type of automotive crash box for the purpose of reducing the weight. Herein, the new type of automotive crash box is designed by variable-thickness rolled blank, the rolling processes of which are shown in Fig. 2 [27]. The variablethickness rolled blank consists of different thickness zones and transition zones, where the transition zones are used to link different thickness zones. As mentioned, directly combining optimization algorithms with finite element analysis will consume a great deal of time to search for the optimal thickness distribution of the new type of automotive crash box. Indeed, highly accurate surrogate models provide an effective way to improve the computational efficiency. From [11], we know that the key issue of constructing highly accurate surrogate models is to obtain representative samples.

The structure of a new type of automotive crash box is shown in Fig. 3, which includes 14 decision variables, i.e., nine different lengths $\left(x_{1}, x_{2}, \ldots, x_{9}\right)$ and five different thicknesses $\left(x_{10}, x_{11}, \ldots, x_{14}\right)$. In addition, there are many constraints. First, each decision variable $x_{i}$ should be in a predefined range, i.e., $\left[x_{i}^{\text {lower }}, x_{i}^{\text {upper }}\right], i=1,2, \ldots, 14$, where $x_{i}^{\text {lower }}$ and $x_{i}^{\text {upper }}$ denote the lower and upper bounds of $x_{i}$, respectively. Secondly, the new type of automotive crash box should satisfy the requirement of the standard industrial size. Therefore, the sum of the length of different thickness zones and transition zones should be equal to a constant $L$. Thirdly, the transition zones should satisfy the 
limitations of manufacturing technology, and thus, the ratio of adjacent thicknesses should be in the range of $[a, b]$. Moreover, the ratio between the difference of adjacent thicknesses and the length of the corresponding transition zone should be in the range of $[c, d]$. Finally, the weight of the new type of automotive crash box should be less than or equal to a constant $M$. The detailed relationships of the decision variables are given in Table I and the parameter values are given in Table II.

From Table I, it can be seen that the experimental domain is subject to 14 boundary constraints, one linear equality constraint, and 17 nonlinear inequality constraints. The ultimate aim of the uniform design of the new type of automotive crash box is to sample uniformly over this constrained experimental domain.

\section{CONCEPTS OF DIFFERENTIAL EVOLUTION}

Differential evolution (DE), proposed by Storn and Price [43], is a population-based optimizer. It is one of the most popular paradigms of evolutionary algorithms and has been successfully applied to solve different kinds of optimization problems [44]-[48]. Suppose that an optimization problem is to minimize the objective function $f(\vec{x})$. Firstly, DE randomly generates $N P$ samples (also called $N P$ individuals) from the decision space:

$$
\vec{x}_{i}=\left(x_{i, 1}, x_{i, 2}, \ldots, x_{i, D}\right) \in S, i=1,2, \ldots, N P
$$

where

- $x_{i, j}(j \in\{1,2, \ldots, D\})$ is the $j$ th decision variable;

- $\quad D$ is the number of decision variables;

- $S=\prod_{j=1}^{D}\left[x_{j}^{\text {lower }}, x_{j}^{\text {upper }}\right]$ is the decision space;

- $x_{j}^{\text {lower }}$ and $x_{j}^{\text {upper }}$ are the lower and upper bounds of $x_{i, j}$, respectively.

These $N P$ samples form the initial population, i.e., an $N P \times D$ matrix. Next, DE adopts three main steps, i.e., mutation, crossover, and selection to evolve the population.

Mutation: By the mutation, DE creates a mutant vector $\vec{v}_{i}=\left(v_{i, 1}, v_{i, 2}, \ldots, v_{i, D}\right)$ for each sample $\vec{x}_{i}$. The two extensively used mutation operators (called DE/rand/1 and DE/currentto-rand/1) are introduced as follows:

- $\mathrm{DE} / \mathrm{rand} / 1$

$$
\vec{v}_{i}=\vec{x}_{r_{1}}+F \times\left(\vec{x}_{r_{2}}-\vec{x}_{r_{3}}\right), i=1,2, \ldots, N P
$$

- DE/current-to-rand/1

$$
\vec{v}_{i}=\vec{x}_{i}+\operatorname{rand} \times\left(\vec{x}_{r_{1}}-\vec{x}_{i}\right)+F \times\left(\vec{x}_{r_{2}}-\vec{x}_{r_{3}}\right), i=1,2, \ldots, N P
$$

where $r_{1}, r_{2}$, and $r_{3}$ are three random and mutually different integers chosen from $[1, N P]$, rand is a uniformly distributed random number from $[0,1]$, and $F \in(0,1]$ is the scaling factor which controls the amplification of the differential vector $\left(\vec{x}_{r_{2}}-\vec{x}_{r_{3}}\right)$.

Crossover: The crossover is implemented on $\vec{x}_{i}$ and its mutant vector $\vec{v}_{i}$ to produce a trial vector $\vec{u}_{i}=\left(u_{i, 1}, u_{i, 2}, \ldots, u_{i, D}\right)$ :

$u_{i, j}=\left\{\begin{array}{ll}v_{i, j} & \text { if } \text { rand }_{j}<C R \text { or } j=j_{\text {rand }} \\ x_{i, j} & \text { otherwise }\end{array}, i=1,2, \ldots, N P, j=1,2, \ldots, D\right.$ where $C R \in[0,1]$ is the crossover control parameter, rand $_{j}$ is the $j$ th evaluation of a uniformly distributed random number between 0 and 1 , and $j_{\text {rand }}$ is an index randomly chosen from $\{1,2, \ldots, D\}$. The condition “ $j=j_{\text {rand }}$ ” ensures that $\vec{u}_{i}$ differs from $\vec{x}_{i}$ by at least one element.

Selection: $\vec{x}_{i}$ is replaced by $\vec{u}_{i}$ if the objective function value of $\vec{x}_{i}$ is not better than that of $\vec{u}_{i}$; otherwise, $\vec{x}_{i}$ will survive into the next generation:

$$
\vec{x}_{i}=\left\{\begin{array}{ll}
\vec{u}_{i}, & \text { if } f\left(\vec{u}_{i}\right) \leq f\left(\vec{x}_{i}\right) \\
\vec{x}_{i}, & \text { otherwise }
\end{array}, i=1,2, \ldots, N P\right.
$$

In DE, the mutation, crossover, and selection are executed generation by generation until a predefined stopping criterion is satisfied. From the introduction, it is evident that DE does not include any complex operators.

\section{A TWO-PHASE DE FOR UNIFORM DESIGNS IN CONSTRAINED EXPERIMENTAL DOMAINS}

\section{A. Fitness Functions}

If a sample satisfies all constraints, then it is called a feasible sample; otherwise, it is called an infeasible sample. A constrained experimental domain is the set of all feasible samples. As pointed out previously, the constrained experimental domain may be a small and irregular region in the decision space because of constraints. The generated samples in the population of DE by the initialization satisfy boundary constraints, but most of them-even all of them-could not satisfy linear/nonlinear and inequality/equality constraints, which indicates that maybe there is no feasible sample in the initial population. Therefore, constraint satisfaction is the first issue for uniform designs in constrained experimental domains.

Based on this analysis, we establish the first fitness function. Since the degree of constraint violation can directly measure the feasibility of a sample, minimizing the degree of constraint violation is an efficient way to motivate a sample toward the constrained experimental domain. The degree of constraint violation of a sample $\vec{x}_{i}(i \in\{1,2, \ldots, N P\})$ on the $k$ th constraint is computed by the following equation:

$$
\begin{cases}G_{k}\left(\vec{x}_{i}\right)=\max \left\{0, g_{k}\left(\vec{x}_{i}\right)\right\}, & 1 \leq k \leq p \\ H_{k}\left(\vec{x}_{i}\right)=\max \left\{0,\left|h_{k}\left(\vec{x}_{i}\right)\right|-\delta\right\}, & 1 \leq k \leq q\end{cases}
$$

where $g_{k}\left(\vec{x}_{i}\right)$ denotes the $k$ th inequality constraint, $p$ is the number of inequality constraints, $G_{k}\left(\vec{x}_{i}\right)$ represents the degree of constraint violation on the $k$ th inequality constraint, $h_{k}\left(\vec{x}_{i}\right)$ denotes the $k$ th equality constraint, $q$ is the number of equality constraints, $\delta$ is the tolerance value to relax the equality constraint to a certain extent, and $H_{k}\left(\vec{x}_{i}\right)$ represents the degree of constraint violation on the $k$ th equality constraint.

Then, the degree of constraint violation of $\vec{x}_{i}$ on all constraints can be expressed as follows:

$$
F F_{1}\left(\vec{x}_{i}\right)=\sum_{k=1}^{p} G_{k}\left(\vec{x}_{i}\right)+\sum_{k=1}^{q} H_{k}\left(\vec{x}_{i}\right)
$$

In this paper, equation (7) is considered to be the first fitness function for the purpose of constraint satisfaction. Note that the smaller the value of $F F_{1}$, the better the performance of a sample. 
Moreover, $F F_{1}\left(\vec{x}_{i}\right)=0$ means that $\vec{x}_{i}$ is a feasible sample. By minimizing equation (7), the degree of constraint violation of the population will decrease gradually, and more and more samples in the population will become feasible. As a result, a predefined number of samples can be obtained in the constrained experimental domain.

The ultimate purpose of uniform designs in constrained experimental domains is to uniformly spread the samples throughout the constrained experimental domain. Therefore, the second issue is related to the uniform distribution of the obtained samples in the constrained experimental domain.

Chen et al. [36] proposed a heuristic method, called discrete particle swarm optimization, for constructing uniform designs in constrained experimental domains. In this method, each individual in the population is an $N P \times D$ array and the central composite discrepancy (CCD) [49] is used to measure the uniformity of an individual. However, it is an NP-hard problem to search for the optimal design based on the CCD criterion. Although a discrete version of CCD can save the computational cost, it is still very time-consuming. For example, the uniform design of the new type of automotive crash box introduced in Section II includes 14 decision variables. If a population consists of 50 samples, then the method in [36] will take about $3 \times 10^{8}$ years to produce the optimal uniform design in the constrained experimental domain. Obviously, it is very hard for this method to be directly applied in practical engineering.

To achieve uniform designs in constrained experimental domains more efficiently, we design a second fitness function, which maximizes the minimum Euclidean distance among the samples in the population. This fitness function is able to make an effective tradeoff between uniform distribution and computational cost, which is implemented as follows.

For each sample $\vec{x}_{i}(i \in\{1,2, \ldots, N P\})$ in the population, we compute the normalized Euclidean distance from $\vec{x}_{i}$ to the other samples, and the minimum normalized Euclidean distance is denoted as min_dis :

$$
\min _{-} d i s_{i}=\min _{j=1,2, \ldots, N P, j \neq i} \operatorname{distance}\left(\operatorname{normalized}\left(\vec{x}_{i}\right) \text {,normalized }\left(\vec{x}_{j}\right)\right)
$$

where distance $(\cdot$,$) represents the Euclidean distance in the$ decision space, and normalized $\left(\vec{x}_{i}\right)$ and normalized $\left(\vec{x}_{j}\right)$ mean that each dimension of $\vec{x}_{i}$ and $\vec{x}_{j}$ is normalized as follows:

$$
\begin{aligned}
& \operatorname{normalized}\left(x_{i, k}\right)=\left(x_{i, k}-x_{k}^{\text {lower }}\right) /\left(x_{k}^{\text {upper }}-x_{k}^{\text {lower }}\right), k=1,2, \ldots, D \\
& \operatorname{normalized}\left(x_{j, k}\right)=\left(x_{j, k}-x_{k}^{\text {lower }}\right) /\left(x_{k}^{\text {upper }}-x_{k}^{\text {lower }}\right), k=1,2, \ldots, D
\end{aligned}
$$

Afterward, the second fitness function can be expressed as follows:

$$
F F_{2}=\min _{i=1,2, \ldots, N P} \min _{-} d i s_{i}
$$

The larger the value of $F F_{2}$, the better the overall uniformity of the population. A smaller value of $F_{2}$ means that some samples in the population are stuck at a sub-region of the constrained experimental domain. On the contrary, a larger value of $\mathrm{FF}_{2}$ means that the samples are distributed relatively uniformly in the constrained experimental domain. The computational time complexity of $F F_{2}$ is $O\left(N P^{2}\right)$.
According to the characteristics of uniform designs in constrained experimental domains and the two fitness functions, we divide the whole evolutionary process into two phases. These two phases are elaborated on next.

\section{B. Phase One}

The first phase aims at obtaining a predefined number of feasible samples by minimizing the first fitness function. Note that the first phase should also provide feasible candidate solutions with good diversity for the next phase to achieve the ultimate purpose, i.e., uniform design. In order to keep the diversity of the population, a possible way is to guide the samples toward the constrained experimental domain from different directions. Inspired by [40], a clustering DE is utilized.

Firstly, population $\boldsymbol{P}$ including $N P$ samples is clustered into $\lfloor N P$ / NS $\rfloor$ subpopulations, where $N S$ denotes the number of samples in each subpopulation and $\lfloor\cdot\rfloor$ denotes rounding down to the nearest integer. Afterward, DE is employed to evolve each subpopulation. The clustering DE is implemented in the following iterative way:

Step 1: Randomly generate a reference point $\vec{r}$ from the decision space and normalize $\vec{r}$ according to equation (9).

Step 2: Set $i=1$ and $\boldsymbol{T P}=\varnothing$. // $\boldsymbol{T P}$ denotes a temporary population

Step 3: Compute the normalized Euclidean distance from $\vec{r}$ to the samples in $\boldsymbol{P}$ and determine the nearest sample in $\boldsymbol{P}$ to $\vec{r}$ (denoted as $\vec{z}$ ).

Step 4: Find $(N S-1)$ samples in $\boldsymbol{P}$, which are nearest to $\vec{z}$. These (NS -1) samples and $\vec{z}$ form a subpopulation, denoted as $\boldsymbol{S P}_{i}$.

Step 5: Delete these NS samples from $\boldsymbol{P}$.

Step 6: Generate an offspring subpopulation (denoted as $\boldsymbol{O P}_{i}$ ) for $\boldsymbol{S P}_{i}$ by the mutation and crossover of $\mathrm{DE}$, and implement the selection of DE on $\boldsymbol{S P}_{i}$ and $\boldsymbol{O P}_{i}$ to update $\boldsymbol{S P}_{i}$. Note that the comparison is based on the first fitness function $F F_{1}$.

Step 7: Incorporate the updated $\boldsymbol{S P}_{i}$ into the temporary population $\boldsymbol{T P}$, i.e., $\boldsymbol{T P}=\boldsymbol{T P} \cup \boldsymbol{S} \boldsymbol{P}_{i}$.

Step 8: $i=i+1$. If $i \leq\lfloor N P$ / NS $\rfloor$, then go to Step 3; otherwise $\boldsymbol{P}=\boldsymbol{P} \cup \boldsymbol{T P}$.

Step 9: If each subpopulation has at least $\left\lfloor N P^{\prime} /(N P / N S)\right\rfloor$ feasible samples, then randomly choose $\left\lfloor N P^{\prime} /(N P / N S)\right\rfloor$ feasible samples from each subpopulation to form a new population $\boldsymbol{P}^{\prime}$ for the second phase; otherwise go to Step 1.

Since different subpopulations converge toward the constrained experimental domain from different directions, in Step 9 we choose $\left\lfloor N P^{\prime} /(N P / N S)\right\rfloor$ feasible samples from each subpopulation to guarantee the diversity of $\boldsymbol{P}^{\prime}$, where $N P^{\prime}$ is the population size of $\boldsymbol{P}^{\prime}$. In general, $N P^{\prime} \ll N P$.

\section{Phase Two}

After the first phase, we obtain the population $\boldsymbol{P}^{\prime}$, which contains a predefined number of feasible samples (i.e., $N P^{\prime}$ ). In 


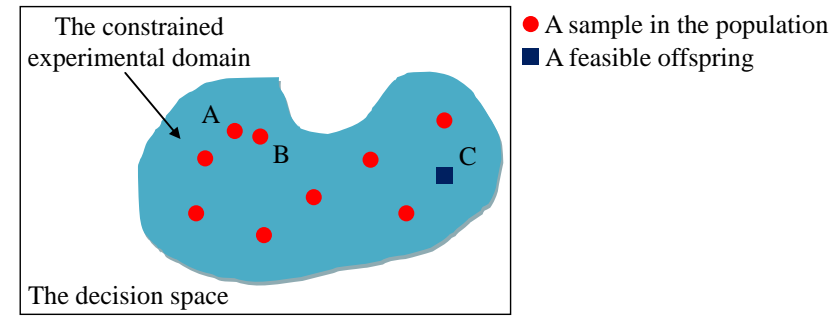

Fig. 4. An example of the replacement strategy. In this figure, $\mathrm{C}$ is a feasible offspring which is added into the population, and $\mathrm{A}$ and $\mathrm{B}$ are two samples in the population. Since A and B have the minimum normalized Euclidean distance and the second minimum normalized Euclidean distance of $\mathrm{A}$ is less than that of $\mathrm{B}, \mathrm{A}$ is deleted from the population. If the updated population has a better value of $F F_{2}$, then the replacement is successful. Otherwise, the population returns to its original state.

the second phase, the mutation and crossover of DE are executed to generate $N P^{\prime}$ offspring for $\boldsymbol{P}^{\prime}$. It is noteworthy that maybe some offspring are not in the constrained experimental domain. Therefore, it is necessary to check whether a newly generated offspring is feasible or not by the first fitness function $F F_{1}$. If all the newly generated offspring are infeasible, DE will be implemented continuously until at least one offspring is feasible.

In order to make $\boldsymbol{P}^{\prime}$ distribute uniformly in the constrained experimental domain, a novel replacement strategy is proposed in this paper. Firstly, the feasible offspring are added to $\boldsymbol{P}^{\prime}$ one by one. After adding one feasible offspring to $\boldsymbol{P}^{\prime}$, we will choose the sample which has the minimum normalized Euclidean distance to the other samples according to equation (8). If several samples have the same minimum normalized Euclidean distance, then we will consider the second minimum normalized Euclidean distance and so forth. At last, we obtain one individual. Subsequently, this individual is deleted from $\boldsymbol{P}^{\prime}$; thus, the population size of $\boldsymbol{P}^{\prime}$ is unchanged. If the updated $\boldsymbol{P}^{\prime}$ has a better value of $F F_{2}$, then the replacement is successful; otherwise, $\boldsymbol{P}^{\prime}$ returns to its original state. If the successful replacement cannot occur over continuous count times, then the second phase halts. Fig. 4 gives a simple example. Through this replacement strategy, the overall uniformity of $\boldsymbol{P}^{\prime}$ can be strengthened constantly during the evolution.

The details of the second phase are given as follows:

Step 1: Compute the fitness function $F F_{2}$ for $\boldsymbol{P}^{\prime}$.

Step 2: Set $k=0$.

Step 3: Generate $N P^{\prime}$ offspring for $\boldsymbol{P}^{\prime}$ by implementing the mutation and crossover of DE.

Step 4: Find the feasible offspring and suppose that there are $N S^{\prime}$ feasible offspring.

Step 5: If $N S^{\prime}=0$, which suggests that there are no feasible offspring, then go to Step 3.

Step 6: For $i=1$ : $N S^{\prime}$

Step 6.1: $\boldsymbol{Q}^{\prime}=\boldsymbol{P}^{\prime}$; // $\boldsymbol{Q}^{\prime}$ denotes the original state of $\boldsymbol{P}^{\prime}$

Step 6.2: Put the $i$ th feasible offspring into $\boldsymbol{P}^{\prime}$;

Step 6.3: Choose the sample having the minimum normalized Euclidean distance according to equation (8) (if several samples have the same minimum normalized
Euclidean distance, then select the sample with the second minimum normalized Euclidean distance and so forth) and delete it from $\boldsymbol{P}^{\prime}$;

Step 6.4: Compute the fitness function $F F_{2}$ for the updated $\boldsymbol{P}^{\prime}$;

Step 6.5: If the updated $\boldsymbol{P}^{\prime}$ has a better value of $F F_{2}$, then it is a successful replacement and $k=0$; otherwise $\boldsymbol{P}^{\prime}=\boldsymbol{Q}^{\prime}$ and $k=k+1$;

Step 6.6: If $k>$ count, break and output $\boldsymbol{P}^{\prime}$;

\section{Step 7: Go to Step 3.}

Remark 1: Based on our introduction, it can be concluded that the above two phases combined with the two fitness functions can not only guide the population toward the constrained experimental domain from different directions quickly, but also have the potential to uniformly scatter the population in the constrained experimental domain.

\section{Performance Criterion}

With the termination of the second phase, the final population $\boldsymbol{P}^{\prime}$ can be obtained. In order to evaluate the performance of $\boldsymbol{P}^{\prime}$, the maximum distance based criterion (called $M D$ ) is adopted [33]. According to [33], firstly we need to produce a test set $\boldsymbol{T S}$, which contains $m$ individuals randomly generated from the constrained experimental domain and $m$ is a very large integer. In this paper, $\boldsymbol{T S}$ is produced by repeatedly implementing the first phase of ToPDE introduced in Section IV-B until $m$ individuals have been obtained in the constrained experimental domain. Afterward, the following procedure is executed:

Step 1: Normalize each sample in $\boldsymbol{P}^{\prime}$ and each individual in TS according to equation (9). After the normalization, let $\boldsymbol{P}^{\prime}=\left\{\vec{x}_{1}^{\prime}, \vec{x}_{2}^{\prime}, \ldots, \vec{x}_{N P^{\prime}}^{\prime}\right\}$ and $\boldsymbol{T S}=\left\{\vec{y}_{1}^{\prime}, \vec{y}_{2}^{\prime}, \ldots, \vec{y}_{m}^{\prime}\right\}$.

Step 2: For each $\vec{y}_{j}^{\prime}(j=1,2, \ldots, m)$ in $T S$, compute the minimum normalized Euclidean distance from $\vec{y}_{j}^{\prime}$ to $\boldsymbol{P}^{\prime}$ : $\min \_\operatorname{dis}\left(\vec{y}_{j}^{\prime}, \boldsymbol{P}^{\prime}\right)=\min _{i=1,2, \ldots, N P^{\prime}} \operatorname{distance}\left(\vec{y}_{j}^{\prime}, \vec{x}_{i}^{\prime}\right)$.

Step 3: Compute the maximum min_dis $\left(\vec{y}_{j}^{\prime}, \boldsymbol{P}^{\prime}\right): M D=$ $\max _{j=1,2, \ldots, m} \min \_d i s\left(\vec{y}_{j}^{\prime}, \boldsymbol{P}^{\prime}\right)$.

In principle, this performance criterion computes the maximum distance between any $\vec{y}_{j}^{\prime}(j=1,2, \ldots, m)$ in $\boldsymbol{T S}$ and the sample in $\boldsymbol{P}^{\prime}$ nearest to $\vec{y}_{j}^{\prime}$. A small value of $M D$ implies that the individuals in $\boldsymbol{T S}$ tend to be close to $\boldsymbol{P}^{\prime}$, and a large value of $M D$ implies that some sub-regions are not well covered by $\boldsymbol{P}^{\prime}$ [33]. Therefore, the smaller the value of $M D$, the better the performance of a method.

\section{E. The Framework of ToPDE}

By integrating the important components introduced in the above four subsections, ToPDE for uniform designs in constrained experimental domains works as follows:

Step 1: Randomly generate an initial population $\boldsymbol{P}$ from the decision space, which contains NP samples; 
TABLE III

The Details of The TWO-Dimensional EXAMPLE

\begin{tabular}{ll}
\hline Relationship of the variables & Type of the constraints \\
\hline$-20 \leq x_{1} \leq 20,-10 \leq x_{2} \leq 10$ & Boundary constraints \\
\hline$-x_{1}+x_{2}-5 \leq 0, x_{1}^{2}+5 x_{2}^{2}-100 \leq 0$, & Inequality constraints \\
$x_{1} x_{2}-10 \leq 0, x_{1} x_{2}+4 \leq 0$ & \\
\hline
\end{tabular}

TABLE IV

PARAMETER SETTINGS OF TOPDE FOR THE TWO-DIMENSIONAL EXAMPLE

\begin{tabular}{cl|ll}
\hline Parameter & Value & Parameter & Value \\
\hline$N P$ & 100 & count & 500 \\
$N S$ & 20 & $F$ & 0.9 \\
$N P^{\prime}$ & 20 & $C R$ & 0.9 \\
\hline
\end{tabular}

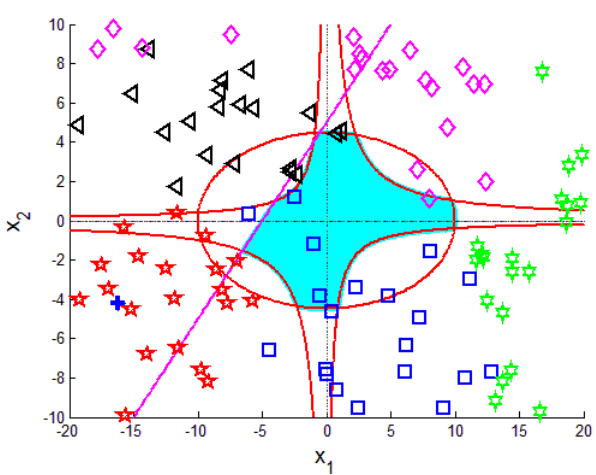

Fig. 5. The initial distribution of the five subpopulations. The blue cross is the reference point, the five subpopulations are depicted with five different colors and shapes, and the cyan part is the constrained experimental domain.

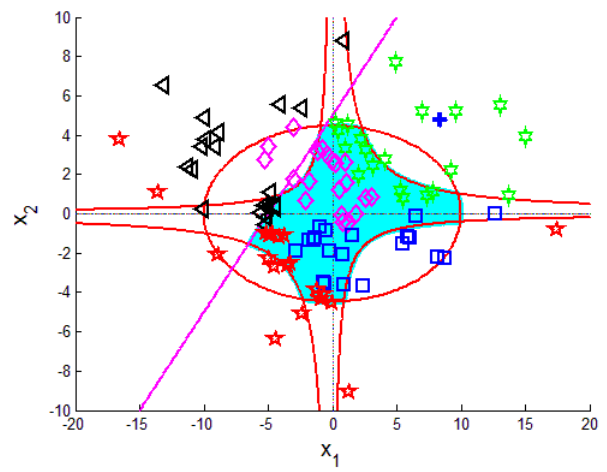

Fig. 6. The end of the first phase. Under this condition, each subpopulation has at least four feasible samples.

Step 2: Implement the first phase and obtain another population $\boldsymbol{P}^{\prime}$ which contains $N P^{\prime}$ diversified samples in the constrained experimental domain;

Step 3: Implement the second phase to make the $N P^{\prime}$ samples in $\boldsymbol{P}^{\prime}$ distribute uniformly in the constrained experimental domain;

Step 4: Evaluate $\boldsymbol{P}^{\prime}$ via the performance criterion.

ToPDE includes six main parameters:

- $N P$ : the size of population $\boldsymbol{P}$ in the first phase;

- $N S$ : the size of each subpopulation in the first phase;

- $N P^{\prime}$ : the size of population $\boldsymbol{P}^{\prime}$ in the second phases;

- count : the maximum tolerance value for the unsuccessful replacement in the second phase;

- $F$ : the scaling factor of DE;

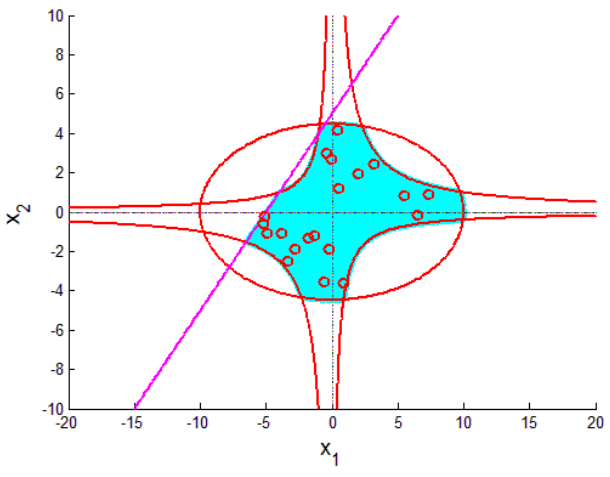

Fig. 7. The initial population of the second phase, which contains 20 feasible samples chosen from the five subpopulations.

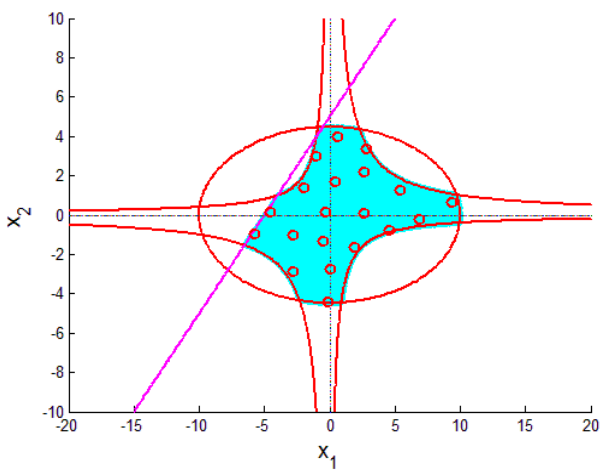

Fig. 8. The final population of the second phase, in which the 20 feasible samples are uniformly distributed in the constrained experimental domain.

TABLE V

THE EXPERIMENTAL RESULTS OF TOPDE OVER 50 RUNS FOR THE TWO-DIMENSIONAL EXAMPLE

\begin{tabular}{cccccccccc}
\hline & $M D$ & & $M D$ & & $M D$ & & $M D$ & & $M D$ \\
\hline 1 & 0.0634 & 11 & 0.0647 & 21 & 0.0584 & 31 & 0.0689 & 41 & 0.0607 \\
\hline 2 & 0.0634 & 12 & 0.0625 & 22 & 0.0585 & 32 & 0.0614 & 42 & 0.0588 \\
\hline 3 & 0.0594 & 13 & 0.0617 & 23 & 0.0609 & 33 & 0.0589 & 43 & 0.0626 \\
\hline 4 & 0.0637 & 14 & 0.0621 & 24 & 0.0651 & 34 & 0.0634 & 44 & 0.0587 \\
\hline 5 & 0.0627 & 15 & 0.0645 & 25 & 0.0603 & 35 & 0.0634 & 45 & 0.0597 \\
\hline 6 & 0.0643 & 16 & 0.0681 & 26 & 0.0581 & 36 & 0.0635 & 46 & 0.0602 \\
\hline 7 & 0.0693 & 17 & 0.1014 & 27 & 0.0772 & 37 & 0.0650 & 47 & 0.0623 \\
\hline 8 & 0.0708 & 18 & 0.0612 & 28 & 0.0577 & 38 & 0.0589 & 48 & 0.0611 \\
\hline 9 & 0.0657 & 19 & 0.0599 & 29 & 0.0646 & 39 & 0.0637 & 49 & 0.0642 \\
\hline 10 & 0.0621 & 20 & 0.0639 & 30 & 0.0636 & 40 & 0.0607 & 50 & 0.0596 \\
\hline
\end{tabular}

Mean Value \pm Std Dev: 0.0635 \pm 0.0066

- $C R$ : the crossover control parameter of DE.

\section{EXPERIMENTAL STUDY}

In this section, at first a two-dimensional example is utilized to show how ToPDE works. Afterward, ToPDE is applied to the uniform design of the new type of automotive crash box. In order to further test the performance of ToPDE, it is also applied to five benchmark test problems. Moreover, the performance of ToPDE is compared with that of another state-of-the-art method, called NTMD [33].

\section{A. A Two-dimensional Example}

A two-dimensional example is designed to explain the working principle of ToPDE. This example contains two decision variables and, therefore, is easy to visualize. In addition, there are one linear inequality constraint and three 
TABLE VI

PARAMETER SETTINGS OF TOPDE FOR THE UNIFORM DESIGN OF THE NEW TYPE OF AUTOMOTIVE CRASH BOX

\begin{tabular}{cl|ll}
\hline Parameter & Value & Parameter & Value \\
\hline$N P$ & 200 & count & 500 \\
$N S$ & 20 & $F$ & 0.9 \\
$N P^{\prime}$ & 100 & $C R$ & 0.9 \\
\hline
\end{tabular}

TABLE VII

THE EXPERIMENTAL RESULTS OF TOPDE OVER 50 RUNS FOR THE UNIFORM DESIGN OF THE NEW TYPE OF AUtOMOTIVE CRASH BOX

\begin{tabular}{cccccccccc}
\hline & $M D$ & & $M D$ & & $M D$ & & $M D$ & & $M D$ \\
\hline 1 & 0.6188 & 11 & 0.5706 & 21 & 0.5490 & 31 & 0.5824 & 41 & 0.5732 \\
\hline 2 & 0.5732 & 12 & 0.6061 & 22 & 0.5919 & 32 & 0.5255 & 42 & 0.5650 \\
\hline 3 & 0.6117 & 13 & 0.5809 & 23 & 0.5818 & 33 & 0.5582 & 43 & 0.5596 \\
\hline 4 & 0.5880 & 14 & 0.5343 & 24 & 0.6042 & 34 & 0.6039 & 44 & 0.6231 \\
\hline 5 & 0.5678 & 15 & 0.5689 & 25 & 0.5352 & 35 & 0.5616 & 45 & 0.5838 \\
\hline 6 & 0.5865 & 16 & 0.5954 & 26 & 0.6042 & 36 & 0.5693 & 46 & 0.5977 \\
\hline 7 & 0.5525 & 17 & 0.6276 & 27 & 0.5339 & 37 & 0.6348 & 47 & 0.5960 \\
\hline 8 & 0.6240 & 18 & 0.5678 & 28 & 0.6165 & 38 & 0.5910 & 48 & 0.6476 \\
\hline 9 & 0.6135 & 19 & 0.5724 & 29 & 0.5585 & 39 & 0.5837 & 49 & 0.6555 \\
\hline 10 & 0.5798 & 20 & 0.5731 & 30 & 0.6051 & 40 & 0.6038 & 50 & 0.5711 \\
\hline \multicolumn{7}{l}{ Mean Value \pm Std Dev: $0.5856 \pm 0.0290$}
\end{tabular}

TABLE VIII

THE EXPERIMENTAL RESULTS OF NTMD OVER 50 RUNS FOR THE UNIFORM DESIGN OF THE NEW TYPE OF AutOMOTIVE CRASH BOX

\begin{tabular}{cccccccccc}
\hline & $M D$ & & $M D$ & & $M D$ & & $M D$ & & $M D$ \\
\hline 1 & 0.7142 & 11 & 0.7472 & 21 & 0.7328 & 31 & 0.6906 & 41 & 0.7619 \\
\hline 2 & 0.7734 & 12 & 0.7673 & 22 & 0.7251 & 32 & 0.7469 & 42 & 0.6448 \\
\hline 3 & 0.6612 & 13 & 0.7267 & 23 & 0.6721 & 33 & 0.6634 & 43 & 0.7216 \\
\hline 4 & 0.7136 & 14 & 0.7313 & 24 & 0.7355 & 34 & 0.7030 & 44 & 0.6883 \\
\hline 5 & 0.6913 & 15 & 0.7610 & 25 & 0.7656 & 35 & 0.7012 & 45 & 0.6646 \\
\hline 6 & 0.6745 & 16 & 0.6935 & 26 & 0.7265 & 36 & 0.7011 & 46 & 0.6983 \\
\hline 7 & 0.7144 & 17 & 0.7547 & 27 & 0.7645 & 37 & 0.7347 & 47 & 0.7274 \\
\hline 8 & 0.7237 & 18 & 0.6924 & 28 & 0.6523 & 38 & 0.7065 & 48 & 0.7185 \\
\hline 9 & 0.6667 & 19 & 0.7063 & 29 & 0.7481 & 39 & 0.6705 & 49 & 0.7013 \\
\hline 10 & 0.7410 & 20 & 0.7597 & 30 & 0.6795 & 40 & 0.7463 & 50 & 0.7382 \\
\hline
\end{tabular}

nonlinear inequality constraints. The details of this example are given in Table III. Assume that the aim is to attain 20 uniform samples in the constrained experimental domain.

The parameter settings of ToPDE are given in Table IV and they were used in the experiments. For the performance criterion, the size of the test set was set to 10,000. After the initialization, the first phase of ToPDE was executed. The aim of this phase is to guide the population toward the constrained experimental domain from different directions promptly. Following the steps of the first phase introduced in Section IV-B, a reference point was randomly selected, and five (i.e., 【NP / NS 」) subpopulations were generated by the clustering DE. The initial distribution of the five subpopulations is shown in Fig. 5. From Fig. 5, we can see that the five subpopulations, depicted with five different colors and shapes, are located at different sub-regions and represent different search directions. If each subpopulation has at least four (i.e., $\left\lfloor N P^{\prime} /(N P / N S)\right\rfloor$ ) feasible samples, then the first phase terminates. The end of the first phase is shown in Fig. 6. Afterward, four feasible samples were randomly chosen from each subpopulation, and these 20 feasible samples formed the initial population of the second phase, which is shown in Fig. 7.

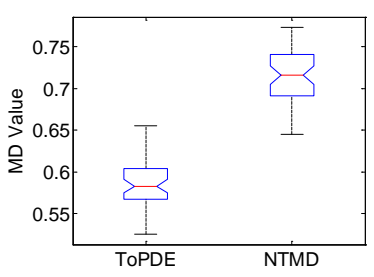

(a)

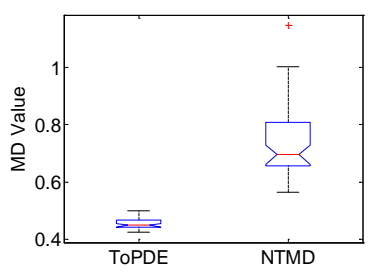

(b)

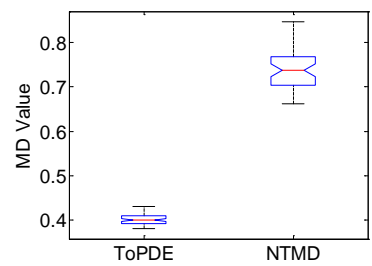

(c)

Fig. 9. Box plots of the experimental results provided by ToPDE and NTMD for the uniform designs of the new type of automotive crash box, G04, and G09. (a) The new type of automotive crash box. (b) G04. (c) G09.

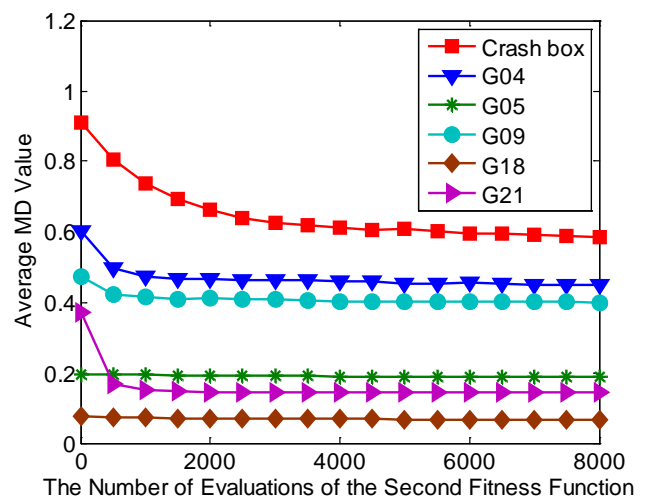

Fig. 10. Evolution of the mean $M D$ values derived from ToPDE over 50 runs versus the number of evaluations of the second fitness function (i.e., $F F_{2}$ ) on the new type of automotive crash box, G04, G05, G09, G18, and G21.

Subsequently, the second phase was triggered, with the purpose of motivating the 20 feasible samples to uniformly cover the constrained experimental domain. The final population with the end of the second phase is shown in Fig. 8. From Fig. 8, it is clear that ToPDE has the capability to uniformly distribute these 20 feasible samples in the constrained experimental domain.

Moreover, we independently ran ToPDE 50 times for this two-dimensional example. The $M D$ values over 50 runs are summarized in Table V. From Table V, it can be seen that ToPDE is able to consistently provide very small $M D$ values, which verifies the stable performance of ToPDE.

\section{B. The Uniform Design of the New Type of Automotive Crash Box}

Section II has introduced a 14-dimensional uniform design of a new type of automotive crash box, the detailed description of which is given in Tables I and II. Assume that the aim is to obtain 100 uniform samples in the constrained experimental domain which is subject to one linear equality constraint and 17 nonlinear inequality constraints.

ToPDE was applied to solve this problem. In order to verify its effectiveness, the performance of ToPDE was compared 
TABLE IX

The DetaILs of G04, G05, G09, G18, AND G21

\begin{tabular}{|c|c|c|c|c|c|c|c|}
\hline Problem & Decision Variable and Constraint & $D$ & $L I$ & $N I$ & $L E$ & $N E$ & $\theta$ \\
\hline G04 & $\begin{aligned} & g_{1}(\vec{x})=85.334407+0.0056858 x_{2} x_{5}+0.0006262 x_{1} x_{4}-0.0022053 x_{3} x_{5}-92 \leq 0 \\
& g_{2}(\vec{x})=-85.334407-0.0056858 x_{2} x_{5}-0.0006262 x_{1} x_{4}+0.0022053 x_{3} x_{5} \leq 0 \\
& g_{3}(\vec{x})=80.51249+0.0071317 x_{2} x_{5}+0.0029955 x_{1} x_{2}+0.0021813 x_{3}^{2}-110 \leq 0 \\
& g_{4}(\vec{x})=-80.51249-0.0071317 x_{2} x_{5}-0.0029955 x_{1} x_{2}-0.0021813 x_{3}^{2}+90 \leq 0 \\
& g_{5}(\vec{x})=9.300961+0.0047026 x_{3} x_{5}+0.0012547 x_{1} x_{3}+0.0019085 x_{3} x_{4}-25 \leq 0 \\
& g_{6}(\vec{x})=-9.300961-0.0047026 x_{3} x_{5}-0.0012547 x_{1} x_{3}-0.0019085 x_{3} x_{4}+20 \leq 0 \\
& 78 \leq x_{1} \leq 102,33 \leq x_{2} \leq 45, \text { and } 27 \leq x_{3}, x_{4}, x_{5} \leq 45\end{aligned}$ & 5 & 0 & 6 & 0 & 0 & $51.1230 \%$ \\
\hline G05 & $\begin{array}{l}g_{1}(\vec{x})=-x_{4}+x_{3}-0.55 \leq 0, g_{2}(\vec{x})=-x_{3}+x_{4}-0.55 \leq 0 \\
h_{1}(\vec{x})=1000 \sin \left(-x_{3}-0.25\right)+1000 \sin \left(-x_{4}-0.25\right)+894.8-x_{1}=0 \\
h_{2}(\vec{x})=1000 \sin \left(x_{3}-0.25\right)+1000 \sin \left(x_{3}-x_{4}-0.25\right)+894.8-x_{2}=0 \\
h_{3}(\vec{x})=1000 \sin \left(x_{4}-0.25\right)+1000 \sin \left(x_{4}-x_{3}-0.25\right)+1294.8=0 \\
0 \leq x_{1} \leq 1200,0 \leq x_{2} \leq 1200,-0.55 \leq x_{3} \leq 0.55, \text { and }-0.55 \leq x_{4} \leq 0.55\end{array}$ & 4 & 2 & 0 & 0 & 3 & $0.0000 \%$ \\
\hline G09 & $\begin{array}{l}g_{1}(\vec{x})=-127+2 x_{1}^{2}+3 x_{2}^{4}+x_{3}+4 x_{4}^{2}+5 x_{5} \leq 0 \\
g_{2}(\vec{x})=-282+7 x_{1}+3 x_{2}+10 x_{3}^{2}+x_{4}-x_{5} \leq 0 \\
g_{3}(\vec{x})=-196+23 x_{1}+x_{2}^{2}+6 x_{6}^{2}-8 x_{7} \leq 0 \\
g_{4}(\vec{x})=4 x_{1}^{2}+x_{2}^{2}-3 x_{1} x_{2}+2 x_{3}^{2}+5 x_{6}-11 x_{7} \leq 0 \\
-10 \leq x_{1}, x_{2}, \ldots, x_{7} \leq 10\end{array}$ & 7 & 0 & 4 & 0 & 0 & $0.5121 \%$ \\
\hline G18 & $\begin{array}{l}g_{1}(\vec{x})=x_{3}^{2}+x_{4}^{2}-1 \leq 0, g_{2}(\vec{x})=x_{9}^{2}-1 \leq 0 \\
g_{3}(\vec{x})=x_{5}^{2}+x_{6}^{2}-1 \leq 0, g_{4}(\vec{x})=x_{1}^{2}+\left(x_{2}-x_{9}\right)^{2}-1 \leq 0 \\
g_{5}(\vec{x})=\left(x_{1}-x_{5}\right)^{2}+\left(x_{2}-x_{6}\right)^{2}-1 \leq 0, g_{6}(\vec{x})=\left(x_{1}-x_{7}\right)^{2}+\left(x_{2}-x_{8}\right)^{2}-1 \leq 0 \\
g_{7}(\vec{x})=\left(x_{3}-x_{5}\right)^{2}+\left(x_{4}-x_{6}\right)^{2}-1 \leq 0, g_{8}(\vec{x})=\left(x_{3}-x_{7}\right)^{2}+\left(x_{4}-x_{8}\right)^{2}-1 \leq 0 \\
g_{9}(\vec{x})=x_{7}^{2}+\left(x_{8}-x_{9}\right)^{2}-1 \leq 0, g_{10}(\vec{x})=x_{2} x_{3}-x_{1} x_{4} \leq 0 \\
g_{11}(\vec{x})=-x_{3} x_{9} \leq 0, g_{12}(\vec{x})=x_{5} x_{9} \leq 0, g_{13}(\vec{x})=x_{6} x_{7}-x_{5} x_{8} \leq 0 \\
-10 \leq x_{1}, x_{2}, \ldots, x_{8} \leq 10 \text { and } 0 \leq x_{9} \leq 20\end{array}$ & 9 & 0 & 13 & 0 & 0 & $0.0000 \%$ \\
\hline G21 & $\begin{array}{l}g_{1}(\vec{x})=-x_{1}+35 x_{2}^{0.6}+35 x_{3}^{0.6} \leq 0 \\
h_{1}(\vec{x})=-300 x_{3}+7500 x_{5}-7500 x_{6}-25 x_{4} x_{5}+25 x_{4} x_{6}+x_{3} x_{4}=0 \\
h_{2}(\vec{x})=100 x_{2}+155.365 x_{4}+2500 x_{7}-x_{2} x_{4}-25 x_{4} x_{7}-15536.5=0 \\
h_{3}(\vec{x})=-x_{5}+\ln \left(-x_{4}+900\right)=0, h_{4}(\vec{x})=-x_{6}+\ln \left(x_{4}+300\right)=0 \\
h_{5}(\vec{x})=-x_{7}+\ln \left(-2 x_{4}+700\right)=0 \\
0 \leq x_{1} \leq 1000,0 \leq x_{2}, x_{3} \leq 40,100 \leq x_{4} \leq 300, \quad 6.3 \leq x_{5} \leq 6.7, \quad 5.9 \leq x_{6} \leq 6.4 \text {, and } \\
4.5 \leq x_{7} \leq 6.25\end{array}$ & 7 & 0 & 1 & 0 & 5 & $0.0000 \%$ \\
\hline
\end{tabular}

with that of NTMD [33]. Previously, NTMD was proposed for the uniform design of a highly constrained mixture experiment which has one linear equality constraint and several inequality constraints. It is worth noting that all the decision variables are involved in the linear equality constraint. The implementation of NTMD is given in the Appendix, in which Step 4 is used to deal with the linear equality constraint and Step 5 is employed to handle other inequality constraints.

Different from [33], some of the decision variables in the uniform design of the new type of automotive crash box are not involved in the linear equality constraint. As shown in Table I, five decision variables (i.e., $x_{10}, x_{11}, \ldots, x_{14}$ ) are not included in the linear equality constraint. Thus, NTMD cannot be directly applied to the uniform design of the new type of automotive crash box. Next, we make a simple revision of Step 4 of NTMD:
Step 4: Produce a number of samples, in which $x_{1}, x_{2}, \ldots, x_{9}$ satisfy the linear equality constraint by implementing equations (14)-(19), and $x_{10}, x_{11}, \ldots, x_{14}$ are uniformly sampled by taking advantage of the Latin square design.

In the experiments, 50 independent runs were implemented for ToPDE and NTMD. The parameter settings of ToPDE are summarized in Table VI. For the performance criterion, the size of the test set was set to 50,000. The experimental results of ToPDE and NTMD are listed in Table VII and Table VIII, respectively.

From Tables VII and VIII, we can see that the mean $M D$ values of ToPDE and NTMD are 0.5856 and 0.7149, respectively. Therefore, the performance of ToPDE is on average $18.09 \%$ better than that of NTMD. Furthermore, Fig. 9(a) provides the box plot of the experimental results of ToPDE 
TABLE X

THE EXPERIMENTAL RESULTS OF TOPDE OVER 50 RUNS ON G04

\begin{tabular}{cccccccccc}
\hline & $M D$ & $M D$ & & $M D$ & & $M D$ & & $M D$ \\
\hline 1 & 0.4813 & 11 & 0.4762 & 21 & 0.4703 & 31 & 0.4405 & 41 & 0.4789 \\
\hline 2 & 0.4455 & 12 & 0.4750 & 22 & 0.4375 & 32 & 0.4866 & 42 & 0.4281 \\
\hline 3 & 0.4310 & 13 & 0.4427 & 23 & 0.4722 & 33 & 0.4465 & 43 & 0.4565 \\
\hline 4 & 0.4454 & 14 & 0.4886 & 24 & 0.4575 & 34 & 0.4449 & 44 & 0.4466 \\
\hline 5 & 0.4406 & 15 & 0.4275 & 25 & 0.4644 & 35 & 0.4414 & 45 & 0.4605 \\
\hline 6 & 0.4369 & 16 & 0.4647 & 26 & 0.4782 & 36 & 0.4353 & 46 & 0.4606 \\
\hline 7 & 0.4643 & 17 & 0.4681 & 27 & 0.4265 & 37 & 0.4784 & 47 & 0.4989 \\
\hline 8 & 0.4331 & 18 & 0.4421 & 28 & 0.4950 & 38 & 0.4459 & 48 & 0.4571 \\
\hline 9 & 0.4246 & 19 & 0.4533 & 29 & 0.4528 & 39 & 0.4502 & 49 & 0.4432 \\
\hline 10 & 0.4465 & 20 & 0.4435 & 30 & 0.4435 & 40 & 0.4310 & 50 & 0.4536 \\
\hline \multicolumn{7}{l}{ Mean Value \pm Std Dev: $0.4543 \pm 0.0190$} & & & \\
\hline \multicolumn{7}{c}{}
\end{tabular}

TABLE XI

THE EXPERIMENTAL RESULTS OF NTMD OVER 50 RUNS ON G04

\begin{tabular}{cccccccccc}
\hline & $M D$ & & $M D$ & & $M D$ & & $M D$ & & $M D$ \\
\hline 1 & 0.6700 & 11 & 0.7938 & 21 & 0.6690 & 31 & 0.6896 & 41 & 0.6230 \\
\hline 2 & 0.7092 & 12 & 0.8518 & 22 & 0.6377 & 32 & 0.5905 & 42 & 0.5777 \\
\hline 3 & 0.7112 & 13 & 0.6836 & 23 & 1.0035 & 33 & 0.7344 & 43 & 0.6838 \\
\hline 4 & 0.6903 & 14 & 0.7976 & 24 & 0.5653 & 34 & 0.8134 & 44 & 0.6965 \\
\hline 5 & 0.6882 & 15 & 0.5988 & 25 & 0.8874 & 35 & 0.8488 & 45 & 0.8155 \\
\hline 6 & 0.8097 & 16 & 0.7760 & 26 & 0.6888 & 36 & 0.6566 & 46 & 0.8581 \\
\hline 7 & 0.7851 & 17 & 0.6961 & 27 & 0.6135 & 37 & 0.6842 & 47 & 0.5957 \\
\hline 8 & 0.7876 & 18 & 0.8370 & 28 & 0.6958 & 38 & 0.5849 & 48 & 0.9569 \\
\hline 9 & 0.6526 & 19 & 0.7081 & 29 & 0.5899 & 39 & 0.7531 & 49 & 0.6053 \\
\hline 10 & 0.8274 & 20 & 1.1475 & 30 & 0.6761 & 40 & 0.6874 & 50 & 0.8443 \\
\hline \multicolumn{7}{l}{ Mean Value \pm Std Dev: $0.7310 \pm 0.1177$} \\
\hline
\end{tabular}

and NTMD. The convergence curve of the average $M D$ value derived from ToPDE over 50 runs is presented in Fig. 10. In Fig. 10 , the iteration will terminate if the number of evaluations of the second fitness function (i.e., $F F_{2}$ ) has reached 8,000. As shown in Fig. 10, ToPDE converges after about 3,000 evaluations of the second fitness function.

The above experimental results reveal that ToPDE is more effective than NTMD for the uniform design of the new type of automotive crash box.

\section{Five Benchmark Test Problems}

In order to further verify the effectiveness of ToPDE on other uniform designs in constrained experimental domains, five benchmark test problems (called G04, G05, G09, G18, and G21) are selected from "the special session and competition on evolutionary constrained real parameter single-objective optimization" of the 2006 IEEE Congress on Evolutionary Computation (IEEE CEC2006) [50]. Note that the purpose of this paper is to study how to uniformly sample over the constrained experimental domain. Consequently, we only exploit the decision variables and constraints of these test problems, and their objective functions are ignored. The decision variables and constraints of G04, G05, G09, G18, and G21 are summarized in Table IX. In Table IX, $D$ is the number of decisions variables, $L I$ is the number of linear inequality constraints, $\mathrm{NI}$ is the number of nonlinear inequality constraints, $L E$ is the number of linear equality constraints, $N E$ is the number of nonlinear equality constraints, and $\theta$ is the estimated feasibility ratio between the constrained experimental domain and the decision space. The characteristics of these five test problems can be briefly summarized as follows:

- G04, G09, and G18 only contain inequality constraints,
TABLE XII

THE EXPERIMENTAL RESULTS OF TOPDE OVER 50 RUNS ON G09

\begin{tabular}{cccccccccc}
\hline & $M D$ & & $M D$ & & $M D$ & & $M D$ & & $M D$ \\
\hline 1 & 0.4013 & 11 & 0.3999 & 21 & 0.3924 & 31 & 0.4070 & 41 & 0.3919 \\
\hline 2 & 0.4094 & 12 & 0.4174 & 22 & 0.3950 & 32 & 0.4072 & 42 & 0.3802 \\
\hline 3 & 0.3987 & 13 & 0.3921 & 23 & 0.3874 & 33 & 0.3978 & 43 & 0.4165 \\
\hline 4 & 0.4309 & 14 & 0.4129 & 24 & 0.3892 & 34 & 0.3836 & 44 & 0.3862 \\
\hline 5 & 0.4060 & 15 & 0.3931 & 25 & 0.3996 & 35 & 0.4179 & 45 & 0.4112 \\
\hline 6 & 0.3879 & 16 & 0.3982 & 26 & 0.3941 & 36 & 0.4091 & 46 & 0.4099 \\
\hline 7 & 0.3911 & 17 & 0.3997 & 27 & 0.4015 & 37 & 0.3988 & 47 & 0.4094 \\
\hline 8 & 0.3810 & 18 & 0.4126 & 28 & 0.4092 & 38 & 0.3925 & 48 & 0.3946 \\
\hline 9 & 0.3883 & 19 & 0.3951 & 29 & 0.4056 & 39 & 0.4145 & 49 & 0.4167 \\
\hline 10 & 0.4167 & 20 & 0.4090 & 30 & 0.3838 & 40 & 0.3893 & 50 & 0.4263 \\
\hline \multicolumn{7}{l}{ Mean Value \pm Std Dev: $0.4012 \pm 0.0120$}
\end{tabular}

TABLE XIII

THE EXPERIMENTAL RESULTS OF NTMD OVER 50 RUNS ON G09

\begin{tabular}{cccccccccc}
\hline & $M D$ & & $M D$ & & $M D$ & & $M D$ & & $M D$ \\
\hline 1 & 0.7430 & 11 & 0.8474 & 21 & 0.6789 & 31 & 0.7315 & 41 & 0.6740 \\
\hline 2 & 0.7603 & 12 & 0.7721 & 22 & 0.7153 & 32 & 0.6705 & 42 & 0.6751 \\
\hline 3 & 0.7075 & 13 & 0.6615 & 23 & 0.6965 & 33 & 0.7353 & 43 & 0.6962 \\
\hline 4 & 0.6724 & 14 & 0.7408 & 24 & 0.7399 & 34 & 0.8025 & 44 & 0.7036 \\
\hline 5 & 0.7249 & 15 & 0.7770 & 25 & 0.7391 & 35 & 0.8350 & 45 & 0.8246 \\
\hline 6 & 0.7648 & 16 & 0.7530 & 26 & 0.7420 & 36 & 0.6949 & 46 & 0.7602 \\
\hline 7 & 0.7291 & 17 & 0.7873 & 27 & 0.7101 & 37 & 0.7721 & 47 & 0.7218 \\
\hline 8 & 0.7618 & 18 & 0.7574 & 28 & 0.7785 & 38 & 0.7114 & 48 & 0.6862 \\
\hline 9 & 0.8189 & 19 & 0.7138 & 29 & 0.7247 & 39 & 0.7685 & 49 & 0.6804 \\
\hline 10 & 0.6844 & 20 & 0.7745 & 30 & 0.6789 & 40 & 0.7221 & 50 & 0.7667 \\
\hline \multicolumn{8}{l}{ Mean Value \pm Std Dev: $0.7382 \pm 0.0460$} \\
\multicolumn{8}{c}{}
\end{tabular}

TABLE XIV

THE AVERAGE AND StandaRd DEVIaTion of the $M D$ VALUES (DENOTED AS “MEAN VALUE” AND “STD DEV”) OBTAINED BY TOPDE AND NTMD OVER 50 RUNS ON G05, G18, AND G21

\begin{tabular}{ccc}
\hline Problem & Method & Mean Value \pm Std Dev \\
\hline \multirow{2}{*}{ G05 } & ToPDE & $0.1869 \pm 0.0648$ \\
& NTMD & $N A$ \\
\hline \multirow{2}{*}{ G18 } & ToPDE & $0.0679 \pm 0.0062$ \\
& NTMD & $N A$ \\
\hline \multirow{2}{*}{ G21 } & ToPDE & $0.1223 \pm 0.0880$ \\
& NTMD & $N A$ \\
\hline
\end{tabular}

and G05 and G21 include both equality and inequality constraints.

- $\quad$ G04 has a moderate feasibility ratio (i.e., 51.1230\%) and G09 has a small feasibility ratio (i.e., $0.5121 \%$ ). In particular, G05, G18, and G21 are highly constrained problems, the feasibility ratios of which are extermely small and approximate to zero ${ }^{1}$.

With respect to these five test problems, 50 independent runs were implemented for ToPDE and NTMD. The parameter settings of ToPDE were the same as in Section V-B. For the performance criterion, the size of the test set was set to 50,000.

\footnotetext{
${ }^{1}$ For a real-world industrial application, maybe an algorithm cannot find any feasible sample. There are two reasons: 1) there does not exist any region satisfying all constraints, and 2) the constrained experimental domain occupies a very small proportion of the decision space, such as G05, G18, and G21. To the best of our knowledge, there is no ready answer for the first scenario. However, for the second scenario, a commonly used method is to relax an equality constraint to a certain degree as in Equation (6). Note that, usually inequality constraints are not relaxed. In Equation (6), $\delta$ is a very small positive number and set to 0.0001 in this paper. This manner slightly enlarges the constrained experimental domain and enables an algorithm to enter the constrained experimental domain more easily. In addition, the power of an optimization algorithm also plays a crucial role in searching for the feasible samples in the second scenario.
} 


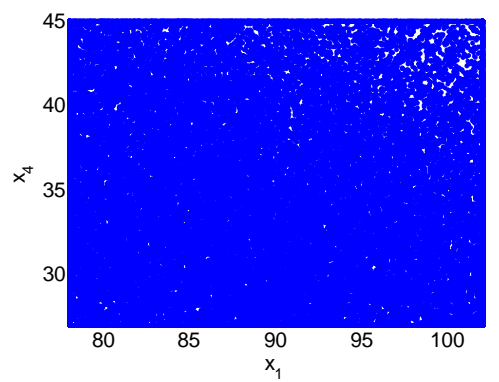

(a)

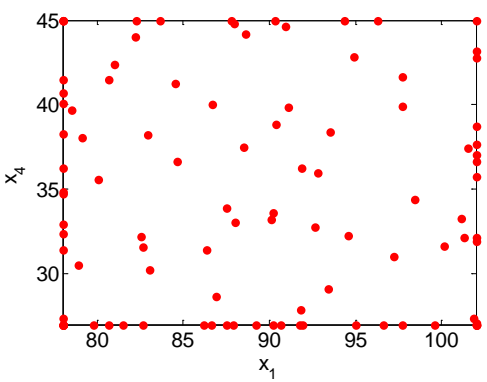

(b)

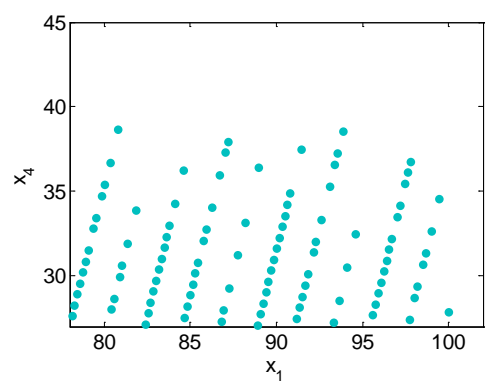

(c)

Fig. 11. The distributions in the $x_{1}-X_{4}$ space on G04. (a) The distribution of the test set containing 50,000 points. (b) The distribution of the final population of ToPDE in a typical run. (c) The distribution of the final population of NTMD in a typical run.

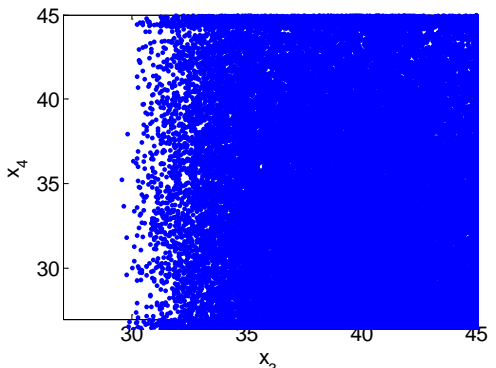

(a)

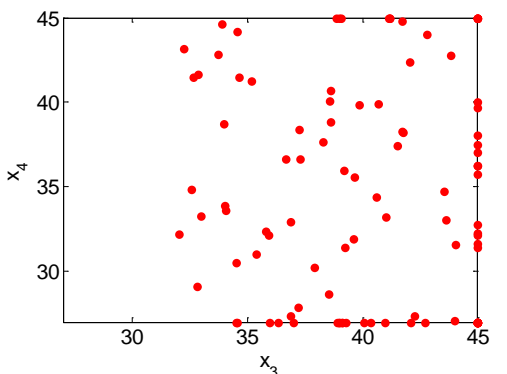

(b)

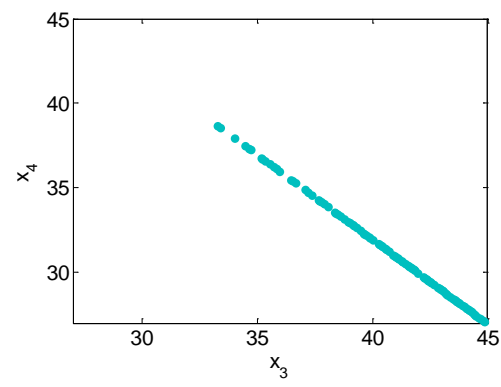

(c)

Fig. 12. The distributions in the $x_{3}-x_{4}$ space on G04. (a) The distribution of the test set containing 50,000 points. (b) The distribution of the final population of ToPDE in a typical run. (c) The distribution of the final population of NTMD in a typical run.

Tables $\mathrm{X}$ and XI summarize the experimental results of ToPDE and NTMD on G04, and Tables XII and XIII summarize the experimental results of ToPDE and NTMD on G09. In addition, the box plots for the performance comparison between ToPDE and NTMD on G04 and G09 are given in Fig. 9(b) and Fig. 9(c), respectively.

Regarding G04, the mean MD values provided by ToPDE and NTMD are 0.4543 and 0.7310 , respectively, which means that ToPDE is on average $37.85 \%$ better than NTMD. Fig. 9(b) describes the distribution of the $M D$ values over 50 independent runs. It is obvious from Fig. 9(b) that the worst $M D$ value derived from ToPDE is even better than the best $M D$ value provided by NTMD.

For G09, the mean MD values of ToPDE and NTMD are 0.4012 and 0.7382 , respectively, which signifies that ToPDE achieves $45.65 \%$ performance improvement. It can be observed again from Fig. 9(c) that the worst $M D$ value of ToPDE is even better than the best $M D$ value of NTMD.

In terms of G05, G18, and G21, the average and standard deviation of the $M D$ values obtained by ToPDE and NTMD over 50 runs are presented in Table XIV. According to our observation, NTMD cannot find any feasible solution even after running one week. Therefore, in Table XIV, “NA” denotes the experimental results of NTMD are not available. The reason why NTMD cannot find any feasible solution on G05, G18, and G21 can be explained as follows. NTMD is very suitable for dealing with one linear equality constraint. However, G05, G18, and G21 do not involve any linear equality constraint. Under this condition, NTMD is equivalent to a traditional uniform design method as pointed out in Section I. Note that when handling other constraints, a traditional uniform design method generates a large number of samples and retains only those samples satisfying all constraints. Therefore, it is essentially a brute-force method. Due to the fact that the feasibility ratios of G05, G18, and G21 are extremely small, it is not difficult to understand that NTMD fails to find any feasible solution. As shown in Table XIV, ToPDE achieves quite good performance since the mean $M D$ values are consistently less than 0.2 .

The convergence curves of the average $M D$ value derived from ToPDE over 50 runs on these five test problems are given in Fig. 10. From Fig. 10, it is interesting to see that ToPDE exhibits very fast convergence speed. In particular, for G05 and G18, the performance of ToPDE is nearly stable at the beginning of the second phase.

The above experimental results demonstrate that ToPDE is also an effective method for other uniform designs in constrained experimental domains, and that ToPDE is not dependent on the type and number of constraints. In the case of moderate and small constrained experimental domains (such as G04 and G09), the performance of ToPDE is significantly better than that of NTMD. Moreover, it seems that NTMD is not capable of coping with the uniform designs in the highly constrained experimental domains without any linear equality constraint (such as G05, G18, and G21).

Remark 2: As pointed out by Borkowski and Piepel [33], for the high-dimensional constrained experimental domain, distance-based methods such as [11], [51], and [52] tend to move samples on or near the boundary, and the interior region is thus not effectively covered. Our proposed ToPDE is also a distance-based method. However, it still works well on the high-dimensional constrained experimental domain. The above phenomenon can be illustrated as follows. Firstly, the methods in [11], [51], and [52] maximize the minimum distance between two samples. It is a local manner to improve the uniformity. 
TABLE XV

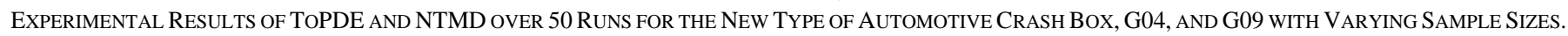

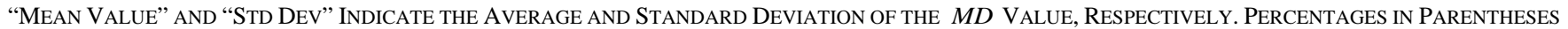
DENOTE THE IMPROVEMENT RATES.

\begin{tabular}{|c|c|c|c|c|c|}
\hline \multirow{3}{*}{ Problem } & \multirow{3}{*}{ Method } & \multicolumn{4}{|c|}{ Sample Size } \\
\hline & & 50 & 100 & 200 & 300 \\
\hline & & $\begin{array}{l}\text { Mean Value } \pm \text { Std Dev } \\
\text { (Improvement Rate) }\end{array}$ & $\begin{array}{l}\text { Mean Value } \pm \text { Std Dev } \\
\text { (Improvement Rate) }\end{array}$ & $\begin{array}{l}\text { Mean Value } \pm \text { Std Dev } \\
\text { (Improvement Rate) }\end{array}$ & $\begin{array}{l}\text { Mean Value } \pm \text { Std Dev } \\
\text { (Improvement Rate) }\end{array}$ \\
\hline \multirow{3}{*}{ Crash box } & ToPDE & $0.6890 \pm 0.0447$ & $0.5856 \pm 0.0290$ & $0.5144 \pm 0.0301$ & $0.4793 \pm 0.0279$ \\
\hline & NTMD & $0.8002 \pm 0.0477$ & $0.7149 \pm 0.0339$ & $0.6457 \pm 0.0297$ & $0.6067 \pm 0.0273$ \\
\hline & & (13.90\%) & (18.09\%) & (20.33\%) & (20.99\%) \\
\hline \multirow{3}{*}{ G04 } & ToPDE & $0.5520 \pm 0.0237$ & $0.4543 \pm 0.0190$ & $0.3782 \pm 0.0106$ & $0.3449 \pm 0.0106$ \\
\hline & NTMD & $0.7380 \pm 0.0753$ & $0.7310 \pm 0.1177$ & $0.6752 \pm 0.1049$ & $0.6312 \pm 0.1148$ \\
\hline & & $(25.20 \%)$ & (37.85\%) & (43.99\%) & $(45.36 \%)$ \\
\hline \multirow{3}{*}{ G09 } & ToPDE & $0.4604 \pm 0.0210$ & $0.4012 \pm 0.0120$ & $0.3512 \pm 0.0150$ & $0.3260 \pm 0.0094$ \\
\hline & NTMD & $1.5780 \pm 0.1270$ & $0.7382 \pm 0.0460$ & $0.6665 \pm 0.0621$ & $0.6201 \pm 0.0527$ \\
\hline & & (70.82\%) & (45.65\%) & (47.31\%) & (47.43\%) \\
\hline
\end{tabular}

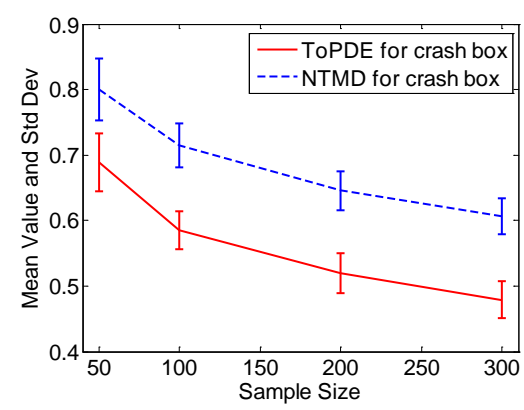

(a)

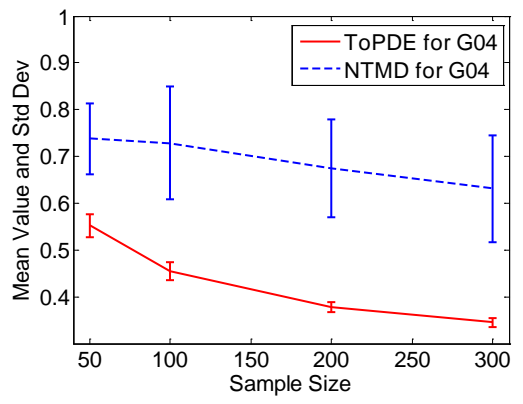

(b)

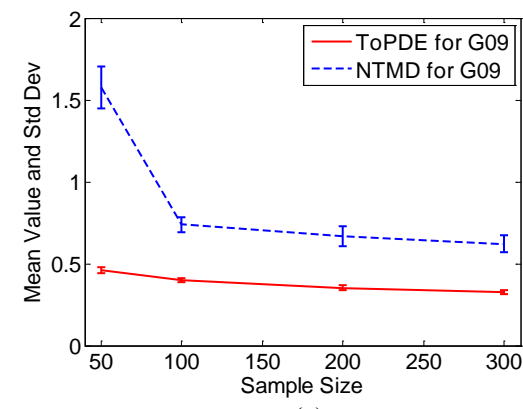

(c)

Fig. 13. The average and standard deviation of the $M D$ values derived from ToPDE and NTMD with four different sample sizes, i.e., 50, 100, 200, and 300. (a) The new type of automotive crash box. (b) G04. (c) G09.

Note that although the performance of these two samples can be improved, the overall uniformity may deteriorate. In contrast, ToPDE maximizes the minimum distance of the entire population in a global manner, which pushes the entire population as far apart as possible. Secondly, the methods in [11], [51], and [52] produce new samples by local search algorithms. As a result, their capability to explore the previously uncovered areas is limited in the high-dimensional constrained experimental domain. On the contrary, ToPDE designs a population-based global search algorithm, which is much more powerful, to produce samples in some promising areas of the high-dimensional constrained experimental domain.

\section{DISCUSSION}

In this section, we investigate the advantage of ToPDE over NTMD, the scalability to the sample size, the effect of the disconnected constrained experimental domain, and the effectiveness of the mutation operator of ToPDE.

\section{A. The Advantage of ToPDE over NTMD}

The experimental results in Sections $\mathrm{V}-\mathrm{B}$ and $\mathrm{V}-\mathrm{C}$ have corroborated the superior performance of ToPDE. In this subsection, the advantage of ToPDE over NTMD is further analyzed by taking G04 as an example. Figs. 11 and 12 show the distributions in the $x_{1}-x_{4}$ space and in the $x_{3}-x_{4}$ space produced by the test set, the final population of ToPDE in a typical run, and the final population of NTMD in a typical run, respectively. In this paper, a typical run means the run in which the $M D$ value ranks 26th (from worst to best) among 50 runs.

As mentioned previously, the general purpose of uniformly sampling over the constrained experimental domain is to provide adequate support for developing the surrogate model. Therefore, it is very important to include the samples on the boundary as well as in the interior of the constrained experimental domain [13], [33]. As shown in Fig. 11, NTMD pays less attention to the boundary of the constrained experimental domain and is very likely to miss some parts of the constrained experimental domain in the $x_{1}-x_{4}$ space. In contrast, ToPDE has the advantage of being able to not only place a number of samples exactly on the boundary of the constrained experimental domain, but also cover the interior of the constrained experimental domain with a lot of samples.

Fig. 12 suggests that ToPDE still maintains good spacefilling performance in the $x_{3}-x_{4}$ space. However, the samples resulting from NTMD are nearly located on a line segment. It is because the nonlinear interaction between $x_{3}$ and $x_{4}$ cannot be identified by NTMD.

\section{B. The Scalability to the Sample Size}

Prior to establishing the surrogate model, a lot of computational resources and costs must be spent evaluating the performance of the obtained samples by physical examples/ simulations. For example, for establishing the surrogate model of a new type of automotive crash box, crashworthiness criteria such as energy absorption and peak force should be evaluated. 


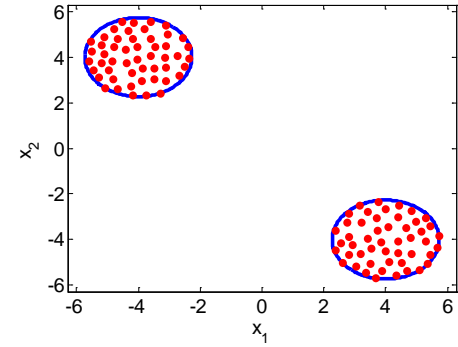

(a)

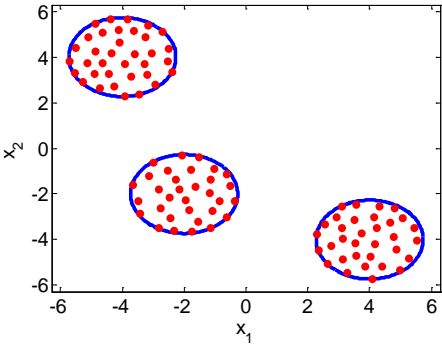

(b)

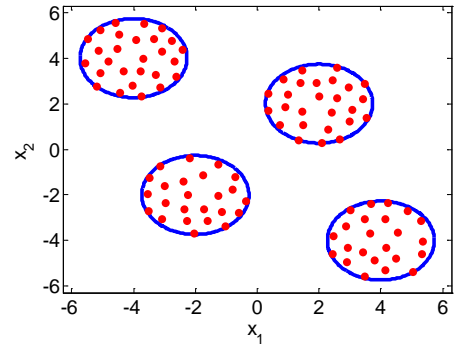

(c)

Fig. 14. The experimental results of ToPDE on three scenarios (denoted as S1-S3) with the disconnected constrained experimental domain defined by equation (12) in a typical run. (a) S1 with two disjointed areas. (b) S2 with three disjointed areas. (c) S3 with four disjointed areas.

Note that this process is computationally expensive; therefore, a moderate sample size is usually recommended (e.g., 100).

However, from the viewpoint of algorithm design, one may still be interested in the scalability of ToPDE and NTMD to the sample size. To this end, we tested four different sample sizes: 50, 100, 200, and 300. The average and standard deviation of the $M D$ values resulting from ToPDE and NTMD are given in Table XV and Fig. 13 presents the visualized comparison. Since NTMD fails to find any feasible solution for G05, G18, and G21, only the experimental result of the new type of automotive crash box, G04, and G09 are summarized in Table $\mathrm{XV}$. It is noteworthy that Table XV also reports the improvement rate achieved by ToPDE against NTMD.

The first observation from Table XV is that the mean value of ToPDE and NTMD gradually decreases with the increase of the sample size. In addition, in terms of the improvement rate, ToPDE has the increasing advantage over NTMD with the increase of the sample size on the three problems, except that for G09 ToPDE achieves the highest improvement rate when the sample size is equal to 50 .

The above comparison verifies that ToPDE consistently outperforms NTMD regardless of the sample size.

\section{The Effect of the Disconnected Constrained Experimental Domain}

In the previous experiments, the constrained experimental domain was connected. A question which arises naturally is whether ToPDE is also applicable to a uniform design in a disconnected constrained experimental domain, which is composed of several disjointed areas. To ascertain the effect of the disconnected constrained experimental domain on the performance of ToPDE, we devised the following test problem:

$$
\begin{aligned}
& g_{1}(\vec{x})=\left(x_{1}+4\right)^{2}+\left(x_{2}-4\right)^{2}-3 \leq 0 \text { or } \\
& g_{2}(\vec{x})=\left(x_{1}-4\right)^{2}+\left(x_{2}+4\right)^{2}-3 \leq 0 \text { or } \\
& g_{3}(\vec{x})=\left(x_{1}+2\right)^{2}+\left(x_{2}+2\right)^{2}-3 \leq 0 \text { or } \\
& g_{4}(\vec{x})=\left(x_{1}-2\right)^{2}+\left(x_{2}-2\right)^{2}-3 \leq 0 \\
& -8 \leq x_{1}, x_{2} \leq 8
\end{aligned}
$$

Based on this test problem, we also designed three scenarios, denoted as S1-S3. S1, S2, and S3 contain the first two constraints, the first three constraints, and all the constraints, respectively. Therefore, their constrained experimental domains respectively consist of two, three, and four disjointed areas, each of which is a circle. In S1-S3, if a sample lies within one of the disjointed areas, then it is feasible.
TABLE XVI

EXPERIMENTAL RESULTS OF TOPDE AND TOPDE_1 OVER 50 RUNS. “MEAN VALUE” AND “STD DEV” INDICATE THE AVERAGE AND STANDARD DEVIATION OF THE MD VALUE, RESPECTIVELY. WiLCOXON's RANK SUM TEST AT A 0.05 SigNIFICANCE LEVEL IS PERFORMED BETWEEN TOPDE AND TOPDE_1.

\begin{tabular}{ccc}
\hline Problem & Method & Mean Value \pm Std Dev \\
\hline \multirow{2}{*}{ Crash box } & ToPDE & $0.5856 \pm 0.0290+$ \\
& ToPDE_1 & $0.6352 \pm 0.0813$ \\
\hline \multirow{2}{*}{ G04 } & ToPDE & $0.4543 \pm 0.0190 \approx$ \\
& ToPDE_1 & $0.4588 \pm 0.0177$ \\
\hline \multirow{2}{*}{ G05 } & ToPDE & $0.1869 \pm 0.0648+$ \\
& ToPDE_1 & $0.3140 \pm 0.1026$ \\
\hline \multirow{2}{*}{ G09 } & ToPDE & $0.4012 \pm 0.0120 \approx$ \\
& ToPDE_1 & $0.4004 \pm 0.0131$ \\
\hline \multirow{2}{*}{ G18 } & ToPDE & $0.0679 \pm 0.0062 \approx$ \\
& ToPDE_1 & $0.0721 \pm 0.0066$ \\
\hline \multirow{2}{*}{ G21 } & ToPDE & $0.1223 \pm 0.0880$ \\
& ToPDE_1 & $N A$ \\
\hline \multirow{2}{*}{ “” and “ $\approx$ " denote that the performance of ToPDE is better than and } \\
\multicolumn{2}{c}{ similar to that of ToPDE_1, respectively }
\end{tabular}

When dealing with the above three scenarios, the parameter settings of ToPDE were kept untouched and the purpose is to obtain 100 uniform samples in each scenario. The experimental results of a typical run provided by ToPDE are given in Fig. 14. As shown in Fig. 14, ToPDE achieves quite promising performance in that a good distribution can be maintained in each disjointed area of each scenario. Moreover, based on our observation, each disjointed area nearly has the same number of samples in each scenario, which implies that ToPDE is also able to maintain a good overall distribution over all the disjointed areas. The excellent performance of ToPDE can be attributed to the fact that the first phase of ToPDE guides the population toward the disconnected constrained experimental domain from different directions and effectively keeps the diversity of the population, and that the second phase of ToPDE facilitates the uniform distribution of samples in each disjointed area.

\section{The Effectiveness of DE/current-to-rand/1 Mutation Operator}

In this paper, DE/current-to-rand/1 is adopted as the mutation operator in DE. To study how the performance of ToPDE is affected by the mutation operator, we implemented a variant of ToPDE called ToPDE_1, in which DE/currentto-rand/1 in equation (3) is replaced by DE/rand/1 in equation (2). The experimental results of ToPDE and ToPDE_1 are presented in Table XVI for the new type of automotive crash 
box, G04, G05, G09, G18, and G21, in which "NA" denotes the experimental results are not available due to the fact that ToPDE_1 cannot enter the second phase on G21.

Table XVI indicates that ToPDE performs statistically better than ToPDE_1 on two problems (i.e., the new type of automotive crash box and G05), and performs similar with ToPDE_1 on three problems (i.e., G04, G09, and G18). However, ToPDE_1 cannot surpass ToPDE even on one problem. More importantly, ToPDE_1 cannot succeed in satisfying the stopping criterion of the first phase on G21. The performance superiority of ToPDE can be explained as follows.

- For DE/rand/1, three individuals are randomly selected from the population for mutation and, consequently, the probability that the offspring is better than the parent is low. Thus, for certain problems (such as G21), based on our observation some subpopulations cannot contain a predefined number of the feasible solutions in the end. In addition, for certain problems (such as the new type of automotive crash box and G05), even though the population can enter the second phase, the replacement cannot occur frequently due to the randomness of DE/ rand/1, which results in the poor performance.

- In contrast, DE/current-to-rand/1 generates the offspring around the current individual. As a result, if the population contains some feasible solutions, then ToPDE is very likely to produce the feasible offspring, thus enhancing search efficiency.

The above discussion suggests that DE/current-to-rand/1 is a more reasonable choice for uniform designs in constrained experimental domains.

Remark 3: In this paper, ToPDE is a framework for uniform designs in constrained experimental domains and DE is its search engine. The function of DE is to generate offspring. The main reason why we chose DE is twofold: 1) the implementation of DE is very simple, and 2) DE provides various mutation operators and we can choose one of them with the most potential to match the characteristics of an optimization problem. Up to now, apart from DE, a considerable number of evolutionary algorithm paradigms have been presented, such as genetic algorithm, evolution strategy, evolutionary programming, particle swarm optimization, artificial bee colony algorithm, and so on. In principle, any evolutionary algorithm paradigm can be incorporated into our framework by replacing DE. It is noteworthy that some evolutionary algorithm paradigms (e.g., particle swarm optimization) make use of the information of the personal best of an individual and/or the entire population's best. In our framework, equation (7) can be utilized to identify the personal best of an individual and the entire population's best in the first phase, and equation (8) can serve a similar purpose in the second phase. In addition, according to the discussion in Section VI-D, generating offspring around the current individuals could also be beneficial to the performance when using other evolutionary algorithm paradigms.

\section{CONCLUSION}

In this paper, a two-phase differential evolution (ToPDE) has been proposed for uniform designs in constrained experimental

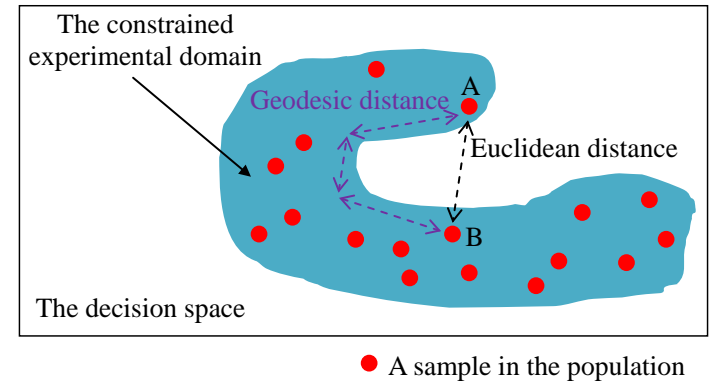

Fig. 15. An example of the geodesic distance and the Euclidean distance between two samples $\mathrm{A}$ and $\mathrm{B}$.

domains. The main task of the first phase is to obtain a predefined number of samples in the constrained experimental domain. For this purpose, a clustering DE has been presented which divides the population into several subpopulations and guides the subpopulations toward the constrained experimental domain from different directions quickly. In addition, the second phase aims at uniformly distributing the feasible samples obtained in the first phase. To accomplish this, a new replacement strategy has been introduced. It is noteworthy that we have also designed two fitness functions for these two phases, respectively. Many experiments have been implemented in this paper. Firstly, we studied the principle of ToPDE by a simple two-dimensional example. Afterward, ToPDE was applied to the uniform designs of a new type of automotive crash box and five benchmark test problems. The performance of ToPDE was compared with that of NTMD, a state-of-the-art method. The experimental results verify the effectiveness of ToPDE.

In this paper, the distance between any two samples in the population is measured by the Euclidean distance. However, the distance indicator depends largely on the type of a uniform design in a constrained experimental domain. Fig. 15 gives an example. In this example, suppose that the geodesic distance is more appropriate than the Euclidean distance. Under this condition, the Euclidean distance will severely underestimate the actual distance between two samples A and B. In the future, we will investigate other types of distance indicator in the realworld applications. Moreover, ToPDE will be applied to more uniform designs in constrained experimental domains of other fields.

The Matlab source code of ToPDE can be downloaded from Y. Wang's homepage: http://ist.csu.edu.cn/YongWang.htm

\section{REFERENCES}

[1] M. Anghileri, L. M. L. Castlletti, F. Invernizzi, and M. Mascheroni, “A survey of numerical models for hail impact analysis using explicit finite element codes,” Int. J. Impact Eng., vol. 31, no. 8, pp. 929-944, 2005.

[2] F. S. Almeida and A. M. Awruch, "Design optimization of composite laminated structures using genetic algorithms and finite element analysis,” Compos. Struct., vol. 88, no. 3, pp. 443-454, 2009.

[3] G. G. Wang and S. Shan, "Review of metamodeling techniques in support of engineering design optimization,” ASME J. Mech. Des., vol. 129, no. 4, pp. 370-380, 2007.

[4] R. J. Yang, N. Wang, C. H. Tho, J. P. Bobineau, and B. P. Wang, "Metamodeling development for vehicle front impact simulation," ASME J. Mech. Des., vol. 127, no. 5, pp. 1014-1020, 2005.

[5] S. Shetty and L. Nilsson, "Multiobjective reliability-based and robust 
design optimization for crashworthiness of a vehicle side impact,” Int. $J$. Veh. Design, vol. 67, no. 4, pp. 347-367, 2015.

[6] T. Ray, M. Asafuddoula, H. K. Singh, and K. Alam, "An approach to identify six sigma robust solutions of multi/many-objective engineering design optimization problems," ASME J. Mech. Des., vol. 137, no. 5, pp. 051404 (14 pages), 2015.

[7] L. Gu, "A comparison of polynomial based regression models in vehicle safety analysis,” In: Proceedings 2011 ASME Design Engineering Technical Conferences - Design Automation Conference, A. Diaz (Ed.), Paper No. DETC2001/DAC-21063, 2011.

[8] J. Fang, G. Sun, N. Qiu, N. H. Kim, and Q. Li, “On design optimization for structural crashworthiness and its state of the art," Struct. Multidisc. Optim., in press, 2016. DOI: 10.1007/s00158-016-1579-y

[9] S. Shan and G. G. Wang, "Survey of modeling and optimization strategies to solve high-dimensional design problems with computationally-expensive black-box functions," Struct. Multidisc. Optim., vol. 41, no. 2, pp. 219-241, 2010.

[10] A. I. J. Forrester and A. J. Keane, "Recent advances in surrogate-based optimization,” Prog. Aerospace Sci., vol. 45, no. 1-3, pp. 50-79, 2009.

[11] E. Stinstra, D. den Hertog, P. Stehouwer, and A. Vestjens, "Constrained maximin designs for computer experiments," Technometrics, vol. 45, no. 4, pp. 340-346, 2003.

[12] D. C. Montgomery. Design and Analysis of Experiments. John Wiley \& Sons, the Eight Edition, 2012.

[13] K. T. Fang and D. K. J. Lin, "Uniform experimental designs and their applications in industry," Handbook of Statistics, vol. 22, pp. 131-170, 2003.

[14] T. W. Simpson, D. K. J. Lin, and W. Chen, "Sampling strategies for computer experiments: design and analysis," International Journal of Reliability and Applications, vol. 2, no.3, pp. 209-240, 2001.

[15] A. A. Giunta, S. F. Wojtkiewicz, and M. S. Eldred, "Overview of modern design of experiments methods for computational simulations," In: The 41st AIAA Aerospace Sciences Meeting and Exhibit, Reno, NV, AIAA, AIAA-2003-0649, 2003.

[16] A. B. Owen, "Orthogonal arrays for computer experiments, integration and visualization," Statistica Sinica, vol. 3, no.1, pp. 439-452, 1993.

[17] A. S. Hedayat, N. J. A. Sloane, and J. Stufken. Orthogonal Arrays: Theory and Applications. Springer Science \& Business Media, Springer, 2012.

[18] L. Franek and X. Jiang, "Orthogonal design of experiments for parameter learning in image segmentation,” Signal Processing, vol. 93, no. 6, pp. 1694-1704, 2013.

[19] J. S. Park, "Optimal Latin-hypercube designs for computer experiments," Journal of Statistical Planning and Inference, vol. 39, no. 1, pp. 95-111, 1994.

[20] H. Dette and A. Pepelyshev, "Generalized Latin hypercube design for computer experiments," Technometrics, vol. 52, no. 4, pp. 421-429, 2012.

[21] B. G. M. Husslage, G. Rennen, E. R. van Dam, and D. D. Hertog, "Space-filling Latin hypercube designs for computer experiments," Optimization and Engineering, vol. 12, no.4, pp. 611-630, 2011.

[22] R. Li, "Model selection for analysis of uniform design and computer experiment," International Journal of Reliability, Quality and Safety Engineering, vol. 9, no. 4, pp. 367-382, 2002.

[23] X. Li, X. Li, and Y. Su, "A hybrid approach combining uniform design and support vector machine to probabilistic tunnel stability assessment," Structural Safety, vol. 61, pp. 22-42, 2016.

[24] Y. Kuo, T. Yang, and B. A. Peters, "Simulation metamodel development using uniform design and neural networks for automated material handling systems in semiconductor wafer fabrication," Simulation Modelling Practice and Theory, vol.15, no. 8, pp. 1002-1015, 2007.

[25] K. T. Fang and Y. Zhang, "Uniform design: Theory and application," Technometrics, vol. 42, no. 3, pp. 237-248, 2000.

[26] S. Golchi and J. L. Loeppky, "Monte Carlo based designs for constrained domains,” 2016. arXiv:1512.07328v2. https://arxiv.org/abs/1512.07328

[27] L. Duan, G. Sun, J. Cui, T. Chen, A. Cheng, and G. Li, "Crashworthiness design of vehicle structure with tailor rolled blank," Struct. Multidiscip. Optim., vol. 53, no. 2, pp. 321-338, 2016.

[28] C. H. Chuang, R. J. Yang, G. Li, K. Mallela, and P. Pothuraju, "Multidisciplinary design optimization on vehicle tailor rolled blank design," Structural and Multidisciplinary Optimization, vol. 35, no. 6, pp. 551-560, 2008.

[29] Z. F. Qi, J. F. Yang, Y. Liu, and M. Q. Liu, "Construction of nearly uniform designs on irregular regions," Communications in Statistics Theory and Methods, in press, 2016. http://dx.doi.org/10.1080/
03610926.2016.1179755

[30] D. Dragulji, T. J. Santner, and A. M. Dean, "Noncollapsing space-filling designs for bounded nonrectangular regions," Technometrics, vol. 54, no. 2, pp. 169-178, 2012.

[31] Y. Z. Liang, K. T. Fang, and Q. S. Xu, "Uniform design and its applications in chemistry and chemical engineering," Chemometrics \& Intelligent Laboratory Systems, vol. 58, no. 1, pp. 43-57, 2001.

[32] L. Zhang, Y. Z. Liang, J. H. Jiang, R. Q. Yu, and K. T. Fang, "Uniform design applied to nonlinear multivariate calibration by ANN," Analytica Chimica Acta, vol. 370, no. 1, pp. 65-77, 1998.

[33] J. J. Borkowski and G. F. Piepel, "Uniform designs for highly constrained mixture experiments," Journal of Quality Technology, vol. 41, no. 1, pp. 35-47, 2009.

[34] Y. Liu and M. Q. Liu, "Construction of uniform designs for mixture experiments with complex constraints," Communications in Statistics Theory and Methods, vol. 45, no. 8, pp. 2172-2180, 2016

[35] K. T. Fang and Z. H. Yang, "On uniform design of experiments with restricted mixtures and generation of uniform distribution on some domains," Statistics \& Probability Letters, vol. 46, no. 2, pp. 113-120, 2000.

[36] R. B. Chen, Y. W. Hsu, Y. Hung, and W. Wang, "Discrete particle swarm optimization for constructing uniform design on irregular regions," Comput. Stat. Date Anal., vol. 72, no. 3, pp. 282-297, 2014.

[37] R. Lekivetz and B. Jones, "Fast flexible space-filling designs for nonrectangular regions," Quality and Reliability Engineering International, vol. 31, no. 5, pp. 829-837, 2015.

[38] E. Benková, R. Harman, and W. G. Müller, "Privacy sets for constrained space-filling," Journal of Statistical Planning and Inference, vol. 171, pp. 1-9, 2016.

[39] K. T. Fang, C. X. Ma, and P. Winker, "Centered $L_{2}$-discrepancy of random sampling and Latin hypercube design, and construction of uniform designs," Mathematics of Computation, vol. 71, pp. 275-296, 2002.

[40] Y. Wang and Z. Cai, "A dynamic hybrid framework for constrained evolutionary optimization,” IEEE Trans. Syst., Man, Cybern. B, Cybern., vol. 42, no. 1, pp. 203-217, 2012.

[41] Y. Shi, P. Zhu, L. Shen, and Z. Lin, "Lightweight design of automotive front side rails with TWB concept," Thin-Walled Structures, vol. 45, no.1, pp. 8-14, 2007.

[42] S. J. Lee, H. A. Lee, S. I. Yi, D. S. Kim, H. W. Yang, and G. J. Park, "Design flow for the crash box in a vehicle to maximize energy absorption," Proceedings of the Institution of Mechanical Engineers, Part D: Journal of Automobile Engineering, pp. 0954407012451545, 2012.

[43] R. Storn and K. Price, "Differential evolution - a simple and efficient heuristic for global optimization over continuous spaces," Journal of Global Optimization, vol. 11, no. 4, pp. 341-359, 1997.

[44] S. Das and P. N. Suganthan, "Differential evolution: a survey of the state-of-the-art," IEEE Transactions on Evolutionary Computation, vol. 15, no. 1, pp. 4-31, 2011.

[45] Y. Wang, Z. Cai, and Q. Zhang, "Differential evolution with composite trial vector generation strategies and control parameters," IEEE Transactions on Evolutionary Computation, vol. 15, no. 1, pp. 55-66, 2011.

[46] Y. Wang, B.-C. Wang, H.-X. Li, and G. G. Yen, "Incorporating objective function information into the feasibility rule for constrained evolutionary optimization," IEEE Transactions on Cybernetics, vol. 46, no. 12, pp. 2938-2952, 2016.

[47] Y. Wang, H.-X. Li, G. G. Yen, and W. Song, "MOMMOP: Multiobjective optimization for locating multiple optimal solutions of multimodal optimization problems," IEEE Transactions on Cybernetics, vol. 45, no. 4, pp. 830-843, 2015.

[48] V. Santucci, M. Baioletti, and A. Milani, "Algebraic differential evolution algorithm for the permutation flowshop scheduling problem with total flowtime criterion," IEEE Transactions on Evolutionary Computation, vol. 20, no. 5, pp. 682-694, 2016.

[49] S. C. Chuang and Y. C. Hung, "Uniform design over general input domains with applications to target region estimation in computer experiments," Comput. Stat. Date Anal., vol. 54, no.1, pp. 219-232, 2010.

[50] J. J. Liang, T. P. Runarsson, E. Mezura-Montes, M. Clerc, P. N. Suganthan, C. A. Coello Coello, and K. Deb, "Problem definitions and evaluation criteria for the CEC 2006 special session on constrained real-parameter optimization,” Technical Report, Nanyang Technological University, Singapore, September 2006. 
[51] W. M. Duckworth, "Some binary maximin distance designs," Journal of Statistical Planning and Inference, vol. 88, no. 1, pp. 149-170, 2000.

[52] M. E. Johnson, L. M. Moore, and D. Ylvisaker, "Minimax and maximin distance designs," Journal of Statistical Planning and Inference, vol. 26, no. 2, pp. 131-148, 1990.

Appendix: The implementation of NTMD [33] for uniform designs in the constrained experimental domains defined by one linear equality constraint and several inequality constraints

It is necessary to point out that in [33] NTMD has two variants. One is the one-pass exchange algorithm and the other is the power-modulo-a-prime algorithm. In this paper, the one-pass exchange algorithm is employed. Moreover, we also make a simple improvement on the setting of $N^{*}$, which gradually increases during the iteration. It is because for a highly constrained problem, NTMD cannot find any feasible solution with small and moderate values of $N^{*}$. In NTMD, the linear equality constraint is formulated as follows:

$$
\sum_{i=1}^{D} x_{i}=1, A_{i} \leq x_{i} \leq B_{i}
$$

where $A_{i}$ and $B_{i}$ are the lower and upper bounds of $x_{i}$, respectively, and $0 \leq A_{i} \leq B_{i} \leq 1$.

NTMD includes the following steps:

Step 1: Given a positive integer $N^{*}$, let $\vec{h}=\left(h_{1}, h_{2}, \ldots, h_{D-1}\right)$ be a vector satisfying the following conditions: 1) $h_{1}<h_{2} \cdots<$ $h_{D-1}<N^{*}$, and 2) $\forall j \in\{1,2, \ldots, D-1\}, \operatorname{gcd}\left(N^{*}, h_{j}\right)=1$, where $\operatorname{gcd}\left(N^{*}, h_{j}\right)$ denotes the greatest common divisor of $N^{*}$ and $h_{j}$. The second condition means that $h_{j}$ is relatively prime $N^{*}$.

$$
\text { Step 2: } c_{i, j}=\bmod \left(i \times h_{j}, N^{*}\right), \quad i=1,2, \ldots, N^{*}, \quad j=1,2, \ldots, D-1,
$$
where $\bmod \left(i \times h_{j}, N^{*}\right)$ denotes the remainder after dividing $i \times h_{j}$ by $N^{*}$. If $c_{i, j}=0$, then reset it to $N^{*}$. The matrix consisting of $c_{i, j}$ is called an $N^{*} \times(D-1)$ lattice-point matrix of integers.

Step 3: $d_{i, j}=\left(2 c_{i, j}-1\right) / 2 N^{*}, \quad i=1,2, \ldots, N^{*}, \quad j=1,2, \ldots, D-1$. Clearly, $0<d_{i, j}<1$ and $\vec{d}_{i}=\left(d_{i, 1}, d_{i, 2}, \ldots, d_{i, D-1}\right)$ is a point in the (D-1)-dimensional unit hypercube.

Step 4: Let $\vec{d}_{i}=\left(0, d_{i, 1}, d_{i, 2}, \ldots, d_{i, D-1}\right)\left(i=1,2, \ldots, N^{*}\right)$. For each $\vec{d}_{i}$, the following equations are calculated:

$$
\begin{gathered}
\Delta_{D}=1 \\
\Delta_{k}=1-\left(d_{i, k}+d_{i, k+1}+\cdots+d_{i, D}\right), k=2,3, \ldots, D \\
e_{k}=\max \left(\frac{A_{k}}{\Delta_{k}}, 1-\frac{B_{1}+B_{2}+\cdots+B_{k-1}}{\Delta_{k}}\right) \\
\phi_{k}=\max \left(\frac{B_{k}}{\Delta_{k}}, 1-\frac{A_{1}+A_{2}+\cdots+A_{k-1}}{\Delta_{k}}\right) \\
x_{i, k}=G\left(d_{i, k}, e_{k}, \phi_{k}, \Delta_{k}, k-1\right) \\
=\Delta_{k}\left\{1-\left[d_{i, k}\left(1-\phi_{k}\right)^{k-1}+\left(1-d_{i, k}\right)\left(1-e_{k}\right)^{k-1}\right]^{1 /(k-1)}\right\} \\
x_{i, 1}=1-\left(x_{i, 2}+x_{i, 3}+\cdots+x_{i, D}\right)
\end{gathered}
$$

After the above process, $\vec{d}_{i}$ is transformed into $\vec{x}_{i}$ which can satisfy the linear equality constraint.
Step 5: Store $\vec{x}_{i}\left(i=1,2, \ldots, N^{*}\right)$ into a predefined set if $\vec{x}_{i}$ satisfies all inequality constraints. If the number of points in the set is larger than or equal to $N P^{\prime}$, i.e., a predefined number of the sample size, then stop; otherwise add the value of $N^{*}$ and go to Step 1 .

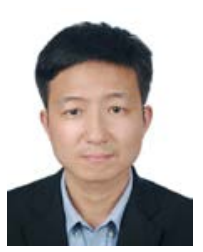

Yong Wang (M'08) received the B.S. degree in automation from the Wuhan Institute of Technology, Wuhan, China, in 2003, and the M.S. degree in pattern recognition and intelligent systems and the Ph.D. degree in control science and engineering both from the Central South University (CSU), Changsha, China, in 2006 and 2011, respectively.

He is currently an Associate Professor with the School of Information Science and Engineering, CSU. His current research interests include the theory, algorithm design, and applications of computational intelligence.

Dr. Wang is a member of the IEEE CIS Task Force on Nature-Inspired Constrained Optimization and the IEEE CIS Task Force on Differential Evolution. He was a reviewer of 50+ international journals and a PC member of $40+$ international conferences. He was awarded the Hong Kong Scholar by the Mainland - Hong Kong Joint Postdoctoral Fellows Program, China, in 2013, the Excellent Doctoral Dissertation by Hunan Province, China, in 2013, the New Century Excellent Talents in University by the Ministry of Education, China, in 2013, the 2015 IEEE Computational Intelligence Society Outstanding PhD Dissertation Award, the Hunan Provincial Natural Science Fund for Distinguished Young Scholars, 2016, and the EU Horizon 2020 Marie Sklodowska-Curie Fellowship, 2016. He is currently serving as an associate editor for the Swarm and Evolutionary Computation.

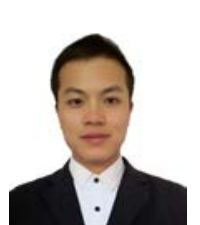

Biao Xu received the B.S. degree in automation from the Hunan Institute of Technology, Hengyang, China, in 2013, and the M.S. Degree in control engineering from the Central South University, Changsha, China, in 2016.

$\mathrm{He}$ is currently an algorithm engineer in Huawei, Shenzhen, China. His current research interests include the algorithm design and applications in telecommunication and computational intelligence.

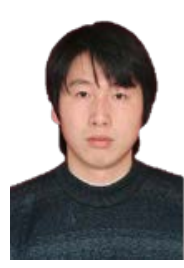

Guangyong Sun received the B.S. and Ph.D. degrees in Mechanical Engineering from Hunan University, Changsha, China, in 2003 and 2011, respectively. He is currently an ARC Discovery Early Career Researcher with the School of Aerospace, Mechanical and Mechatronic Engineering, Faculty of Engineering, The University of Sydney. His research interests include lightweight design, structural optimization, and automotive safety.

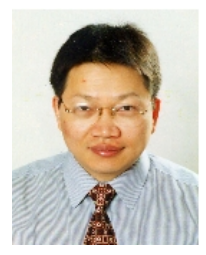

Shengxiang Yang (M'00--SM'14) received the B.Sc. and M.Sc. degrees in automatic control and the Ph.D. degree in systems engineering from Northeastern University, Shenyang, China in 1993, 1996, and 1999, respectively.

He is currently a Professor in Computational Intelligence and Director of the Centre for Computational Intelligence, School of Computer Science and Informatics, De Montfort University, Leicester, U.K.

He has over 220 publications. His current research interests include evolutionary computation, swarm intelligence, computational intelligence in dynamic and uncertain environments, artificial neural networks for scheduling, and relevant real-world applications. He serves as an Associate Editor or Editorial Board Member of 8 international journals, such as the IEEE Transactions on Cybernetics, Information Sciences, Evolutionary Computation, Neurocomputing, and Soft Computing. 